\title{
A paradigm shift of PD-L1 immunotherapy based on a tracking-to-triggering immunoediting effect of 2- $\left[{ }^{18} \mathrm{~F}\right] \mathrm{FDG}$
}

\section{Xuejun Wen}

Xiamen University

Changrong Shi

Xiamen University

\section{Xinying Zeng}

Xiamen University

\section{Liang Zhao}

The First Affiliated Hospital of Xiamen University

\section{Lanlin Yao}

The First Affiliated Hospital of Xiamen University

\section{Lixia Feng}

Xiamen University

\section{Jinxiong Huang}

The First Affiliated Hospital of Xiamen University

\section{Yesen Li}

The First Affiliated Hospital of Xiamen University

\section{Qin Lin}

The First Affiliated Hospital of Xiamen University

\section{Haojun Chen}

The First Affiliated Hospital of Xiamen University

\section{Rongqiang Zhuang}

State Key Laboratory of Molecular Vaccinology and Molecular Diagnostics \& Center for Molecular Imaging and Translational Medicine, School of Public Health, Xiamen University, Xiamen

\section{Xiaoyuan Chen}

National University of Singapore

\section{Xianzhong Zhang}

Xiamen University https://orcid.org/0000-0001-8591-8301

\section{Zhide Guo ( $\sim$ gzd666888@xmu.edu.cn )}

Xiamen University https://orcid.org/0000-0002-9110-159X 
Article

Keywords: Immune Checkpoint Blocker, Radiopharmaceuticals, Immune-favorable Microenvironment, Tumor Immunotherapy, Radiotracer Stimulation

Posted Date: February 23rd, 2021

DOI: https://doi.org/10.21203/rs.3.rs-221828/v1

License: (c) (i) This work is licensed under a Creative Commons Attribution 4.0 International License.

Read Full License 


\section{Abstract}

Efforts have been devoted to select eligible candidates for PD-1/PD-L1 immune checkpoint blocker (ICB) immunotherapy based on radiopharmaceuticals. Here, we have observed a tracking-to-triggering (referred to as "T2T") immunoediting effect of the employed radionuclides. In particular, we found the usefulness of 2-[ $\left.{ }^{18} \mathrm{~F}\right] \mathrm{FDG}$ in cancer ICB therapy. Given that the PD-L1 expression is upregulated after the administration of various radiotracers, the predictive result from PET/SPECT imaging should be treated with caution. Viewed positively, radiotracers are potential immunomodulators to create an immunefavorable microenvironment for tumor immunotherapy. Improving aPD-L1 mAb utilization and significant tumor growth delay are observed when the personalized therapeutic alliance of radiotracer stimulation and ICB are employed. This new paradigm has the potential to expand the traditional tumor theranostic model and implement precision cancer immunotherapy.

\section{Introduction}

The great success of immunotherapy has initiated a new phase in cancer treatment, and an increased understanding of mechanisms leads to the development of various immune checkpoint blockers (ICBs). ICB antibodies targeting the immune checkpoint programmed death receptor 1 (PD-1) expressed on Tcells and its immune regulatory ligand PD-L1 have revolutionized the oncology ${ }^{1,2}$. Unfortunately, immunotherapy with PD-1/PD-L1 ICBs is less pronounced in high percentage of malignancies. There is a broad consensus that targeting PD-L1 is not equally successful in PD-1/PD-L1 ICB immunotherapy and should have tumor PD-L1 expression as a prerequisite. Thereby, tumor PD-L1 expression is deemed to be a predictive biomarker for pinpointing potential candidates who might benefit most from PD-1/PD-L1 immunotherapy ${ }^{3,4}$.

Early studies used ex vivo immunohistochemical (IHC) staining to evaluate PD-1/PD-L1 status, which was affected by different antibodies, varying protocols, scoring systems and positive/negative staining thresholds ${ }^{5-7}$. Moreover, repetitive tumor sample immune infiltration quantifications during therapy monitoring are impractical, highlighting the necessity of developing convenient and reliable PD-L1 testing methods. The ability to accurately visualize PD-L1 expression by non-invasive positron emission tomography (PET) or single-photon emission computed tomography (SPECT) imaging would offer new insights by evaluating the immune status throughout the body at multiple time points longitudinally. Typically, glucose analog $\left[{ }^{18} \mathrm{~F}\right]$ fluorodeoxyglucose $\left(2-\left[{ }^{18} \mathrm{~F}\right] \mathrm{FDG}\right)$, a most commonly prescribed radiotracer in nuclear medicine, continues to occupy center stage in the clinical routine. As tumor cells and activated immune cells (such as T cells) usually present specific glucose metabolism, whole-body noninvasive imaging of 2-[ $\left.{ }^{18} \mathrm{~F}\right] \mathrm{FDG}$ could help predict or monitor the response of ICB immunotherapy ${ }^{8-10}$. It is also worth noting that a wide variety of PD-L1-targeting PET/SPECT radiotracers have been evaluated for clinical translation to select eligible candidates for anti-PD-1/PD-L1 immunotherapies, which show the opportunity for either replacing or complementing 2-[18 F]FDG scanning in the immunoimaging ${ }^{11-16}$. 
In this study, we tested $2-\left[{ }^{18} \mathrm{~F}\right] \mathrm{FDG}$, as an early immunomodulator, to remodel the PD-L1 expression. We demonstrate for the first time to our knowledge, across multiple tumor cells and mouse models including patient-derived xenografts (PDXs) derived from NSCLC, that 2-[18 F]FDG-based PET imaging may result in deceitful and temporary upregulation of PD-L1 expression. This means the role of $2-\left[{ }^{18} \mathrm{~F}\right] \mathrm{FDG}$ for tumor immune microenvironment (TIME) is much more like an immunomodulator than an indicator. Although controversial, with growing evidences ${ }^{17,18}$ that upregulation of PD-L1 expression is beneficial to PD-1/PDL1 immune modulation therapy, we would like to see if imaging radiotracer is a reliable coagent for building an immune-favorable microenvironment for enhancing the efficacy of anti-PD-L1.

\section{Results}

\section{Tumor PD-L1 expression is upregulated after radionuclide or radiotracer stimulation in vitro}

First, we validated the radionuclide-induced PD-L1 upregulation in multiple tumor cell lines. For quick reference, Fig. $1 \mathrm{~A}$ lists the radionuclides used in this article. ${ }^{18} \mathrm{~F},{ }^{99 \mathrm{~m}} \mathrm{Tc},{ }^{177} \mathrm{Lu},{ }^{64} \mathrm{Cu}$ and ${ }^{131}$ I were compared on multiple tumor cell lines (melanoma, breast and colorectal cancer cells) in the immunofluorescence assay (Fig. 1B), which revealed that different radionuclides upregulated PD-L1 expression to different degrees. This stimulation was also embodied prominently through the flow cytometric analysis. As shown in Fig. S1, the proportions of PD-L1-positive cells in the CT26, MC38, 4T1 and B16F10 tumor cells were significantly increased after co-incubation with radionuclides.

Taking ${ }^{18} \mathrm{~F}$ for instance, the percentages of PD-L1 positive cells increased from $23.3 \%$ to $96.5 \%, 54.3 \%$ to $98.7 \%, 21.8 \%$ to $60.6 \%$, and $61.4 \%$ to $96.2 \%$ in the CT26, MC38, $4 \mathrm{~T} 1$ and B16F10 tumor cells after $24 \mathrm{~h}$, respectively. Heat maps generated from reverse transcription-quantitative real-time PCR (RT-qPCR) analysis (Fig. 1C) revealed that all the radionuclides increased the expression of PD-L1 mRNA on tumor cells. For example, after $8 \mathrm{~h}$ co-incubation, ${ }^{18} \mathrm{~F}$ increased PD-L1 mRNA on MC38 and CT26 cells by 53fold and 17-fold, respectively. For radiotherapeutic isotopes, ${ }^{64} \mathrm{Cu}$ increased PD-L1 mRNA on MC38 cell by 28-fold, and ${ }^{177}$ Lu increased PD-L1 mRNA on CT26 cell by 22-fold.

The expression of PD-L1 was elevated to a greater extent by a higher dose of radiotracer, which was further confirmed by flow cytometric analysis in Fig. 1D, clearly indicating that PD-L1 was upregulated in a dose-dependent manner. The expression levels of PD-L1 mRNA and protein in MC38 and CT26 cell lines after stimulation with 2-[18 F]FDG were further evaluated by Western blot (WB) (Fig. 1E). As expected, PDL1 expression was significantly increased in response to radionuclides.

\section{Differentially expressed genes (DEGs) and potential mechanisms of radionuclide-induced PD-L1 upregulation}

Transcriptomic analysis and WB study were performed to explore the potential mechanism of PD-L1 upregulation stimulated by radionuclides. From the volcano plot (Fig. 1F), there were a total of 2002 
DEGs which had changed in 2-[18 $\mathrm{F}]$ FDG-treated MC38 cells compared to the control group, with 1223 upregulated genes and 779 downregulated genes $\left(\left|\log _{2}(\mathrm{FC})\right|>1.0, P\right.$-value $\left.<0.05\right)$. For $2-\left[{ }^{18} \mathrm{~F}\right] \mathrm{FDG}$-treated CT26 tumor cells, the changed number was 2167 (1357 upregulated genes and 810 downregulated genes).

A total of 21725 genes and 21144 genes were identified in 2-[ $\left[{ }^{18} \mathrm{~F}\right] \mathrm{FDG}$ treated MC38 cells and CT26 cells, respectively. As shown in Fig. 1G, Fos, Stat3, Nfkbia, Nfkbib, Nfkbie and Cd274 (PD-L1) genes in 2$\left[{ }^{18} \mathrm{~F}\right] \mathrm{FDG}$ treated MC38 cells were significantly upregulated compared with the untreated cells. Note that Nfkbia, Nfkbib and Nfkbie genes belong to the NF-kappa-B (NF-KB) inhibitor family, which has been reported to upregulate PD-L1 transcription in tumor cells, such as ovarian cancer, gastric carcinoma and lung cancer ${ }^{19-21}$. As verified in previous studies ${ }^{22,23}$, the IKBa kinases (IKK) is a key regulator of the NF-KB pathway and TANK-binding kinase 1 (TBK1) is closely related to the phosphorylation of IRF3.

We also showed the similar results that the radionuclide-induced PD-L1 upregulation was positively correlated with phosphorylated NF-KB P65 (p-NF-KB P65) and phosphorylated IRF3 (p-IRF-3) in radionuclide-treated MC38 cells $($ Fig. $1 \mathrm{H})$. Intriguingly, we found that the PD-L1 upregulation in radionuclide-treated MC38 cells could be blocked by the inhibitors of IKK or TBK1. All these data suggested that the activation and phosphorylation of NF-KB and IRF3 have contributed to promoting PDL1 expression in radiation-induced MC38 murine colon carcinoma cells.

DEGs were mapped into the Kyoto Encyclopedia of Genes and Genomes (KEGG) pathway database to further explain the individual function analysis. Herein, transcriptome analysis using the RNA-seq technology was applied to compare DEGs between 2-[18 F]FDG and saline-treated MC38 cells. As shown in Fig. 11, many receptor-interaction signaling pathways and metabolic pathways were significantly enhanced, including the cytokine-cytokine receptor interaction, the NOD-like receptor signaling pathway, necroptosis, TNF signaling pathway, IL-17 signaling pathway, and the Jak-STAT signaling pathway. According to the literature reports, the NF-KB signaling pathway is one of the major NOD-like receptor signaling pathways, that Nod1 and Nod2 stimulation induces NF-KB activation ${ }^{24}$. Besides, TNF (also known as TNF-a) could induce the expression of NF-KB target genes and trigger the activation of NF-KB signaling pathway, which indirectly upregulated PD-L1 expression ${ }^{25,26}$. These results suggest that multiple inflammatory signaling pathways and metabolic process participate in 2-[ $\left[{ }^{18} \mathrm{~F}\right] \mathrm{FDG}$ induced MC38 cells.

\section{Radiotracers cause PD-L1 upregulation in tumor tissue}

With the help of the IHC technique, PD-L1 expression in tumor tissue was compared between different groups. We first investigated the biodistribution profile of 2-[18 F]FDG and ${ }^{131}$ I-aPD-L1 in CT26 and MC38 tumor types at different time points using a small-animal PET scanner. The signals in the tumor sites were well delineated from that of other tissues, indicating the high affinity of the radiotracers to tumor 
lesion (Fig. 2A). Strikingly, as shown in Fig. 2B,C, PD-L1 levels in the tumor region with radiotracers uptake were more strongly positive compared to control samples.

IFN- $y$ and active $\mathrm{CD} 4^{+} / \mathrm{CD} 8^{+} \mathrm{T}$ cells in TIME play important roles in mediating antitumor immunity. For this reason, we used 2-[ $\left.{ }^{18} \mathrm{~F}\right] \mathrm{FDG}$ as a stimulus to observe the responses of TIME and determine the optimized immunotherapy time window for the administration of aPD-L1 mAb. From the IHC in Fig. 2D,E, we could see that $2-\left[{ }^{18} \mathrm{~F}\right] \mathrm{FDG}$ gradually upregulated the expression of IFN- $\gamma$, and enhanced the infiltration of $\mathrm{CD} 4^{+}$and $\mathrm{CD} 8^{+} \mathrm{T}$ cells over time. Prior studies have demonstrated that the expression of PD-L1 has been an inclusion criterion for selecting patients of non-small cell lung cancer (NSCLC) for anti-PD-L1 treatment ${ }^{27,28}$. To date, PD-1/PD-L1 ICBs have shown promise in advanced NSCLC without driver oncogene mutations, but wider use is restricted to the low objective response rate ${ }^{29,30}$. In this study, we established NSCLC-PDX models to clarify this T2T immunoediting effect further. After 2- $\left[{ }^{18} \mathrm{~F}\right] \mathrm{FDG}$ PET imaging (Fig. 3A,B), the PDXs were divided into high and low 2-[18 F]FDG uptake (denoted as PDX and $\mathrm{PDX} \mathrm{L}_{\mathrm{LFDG}}$ ) groups to evaluate the in vivo biological behavior of radiotracers and predict the PD-L1 response of NSCLC to the radionuclide. Flow cytometry analysis revealed that the PD-L1 positive population in $\mathrm{PDX}_{\mathrm{L-FDG}}(24.9 \pm 3.5 \%)$ was lower than that in $\mathrm{PDX}_{\mathrm{H}-\mathrm{FDG}}(76.9 \pm 4.9 \%)$ at $4 \mathrm{~h}$ p.i. $($ Fig. 3C,D).

For PDX $X_{L-F D G}$ tumor, PD-L1 expression increased from $16.2 \pm 2.3 \%$ (injected with saline) to $24.9 \pm 3.5 \%$ (injected with 2-[ $\left.{ }^{8} \mathrm{~F}\right] \mathrm{FDG}$ ) at $4 \mathrm{~h}$ p.i., $\left.{ }^{*} p \leq 0.05\right)$. For $\mathrm{PDX}{ }_{\mathrm{H}-\mathrm{FDG}}$ tumor, this uptrend was even more noticeable (from $40.1 \pm 6.5 \%$ to $76.9 \pm 4.9 \%,{ }^{\star \star} p \leq 0.01$ ). That is probably means improved PD-L1 expression in 2-[18 F]FDG groups was associated with the stimulation of ${ }^{18} \mathrm{~F}$. Consistent with flow cytometry results, PD-L1 IHC showed more prominent expression in post-tracer PDX $\mathrm{H}_{\mathrm{H}-\mathrm{FDG}}$ tumor biopsies (Fig. 3E). Hence, as we have observed, the T2T effect of radionuclides could reasonably explain the PDL1 upregulation in the tumor.

\section{$2-\left[{ }^{18} \mathrm{~F}\right] \mathrm{FDG}$ causes increased aPD-L1 uptake in tumor}

Intuitively, the PD-L1 upregulation caused by radiotracer would increase aPD-L1 mAb uptake in the tumor. We confirmed this with a fluorescent APD-L1 probe (Fig. 4A). The flow cytometric analysis showed that 2-

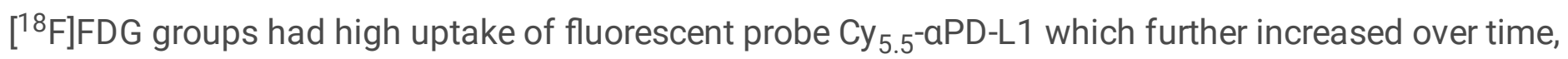
presumably due to the upregulated PD-L1 levels in CT26 and MC38 tumor cells. In striking contrast, much lower $\mathrm{Cy}_{5.5^{-}}$aPD-L1 uptakes were observed in the control tumors without 2-[ $\left.{ }^{18} \mathrm{~F}\right] \mathrm{FDG}$ treatment. Representative histograms of the PD-L1 expression after radionuclides stimulation were shown in Fig. 4B.

\section{T2T effect sensitizes the TIME to immunotherapy and enhances the immunological memory}


To further explore the T2T potential of radiotracers for enhancing immunotherapy, we subsequently investigated the effect of APD-L1 mAb on MC38 tumor growth delay in cooperation with $2-\left[{ }^{18} \mathrm{~F}\right] \mathrm{FDG}$. As shown in Fig. 5A and Fig. S2A, tumor models were treated with either 2-[18 F]FDG, aPD-L1 mAb, or their combination in specific treatment sequences. In the combination groups, aPD-L1 mAb was tail vein injected into the tumor-bearing mice at different intervals (simultaneous injection, 4-h and 24-h; hereinafter referred as @ 4 h and @ 24 h) after administration of the radiotracer. Fig. 5B,C and Fig. S2B illustrated the tumor volumes, time-dependent tumor growth curves, weight changes and survival curves for each group. In the control groups of APD-L1 mAb and saline alone, the tumor sizes developed uncontrollably. Also, single-administration of 2-[ $\left.{ }^{18} \mathrm{~F}\right] \mathrm{FDG}$ did not significantly alter MC38 tumor growth. We then compared the therapeutic effect of aPD-L1 mAb which was administered simultaneously, $4 \mathrm{~h}$ or $24 \mathrm{~h}$ post radiotracer injection. Notably, the 4 -h interval turned out to be the most optimal treatment sequence, and administration of $37 \mathrm{MBq} 2-\left[{ }^{18} \mathrm{~F}\right] \mathrm{FDG}+400 \mathrm{mg}$ aPD-L1 mAb @ 4 h resulted in the maximum therapeutic efficacy ( $5 / 8$ of the tumor mice were completely cured), clearly indicating that the T2T antitumor immunotherapy was regulated in a dose and time-dependent manner. As shown in Fig. 5D, we performed 2-[ $\left.{ }^{18} \mathrm{~F}\right] \mathrm{FDG}-\mathrm{PET}$ imaging on day 0 and day 90 to provide visualization for evaluating therapeutic effect in the best-performing group. Moreover, the body weights of mice were almost identical for all groups during the therapy period (Fig. 5C) and no obvious side effects were observed in the fully recovered mice (Fig. S3), indicating that the T2T antitumor therapeutic strategy was well tolerated.

To verify the immunological memory of T2T-based immune checkpoint therapy, the effector memory $T$ $\left(\mathrm{T}_{\mathrm{EM}}\right)$ cells $\left(\mathrm{CD} 8{ }^{+} \mathrm{CD} 44^{+} \mathrm{CD} 62 \mathrm{~L}^{-}\right.$and $\left.\mathrm{CD} 4^{+} \mathrm{CD} 44^{+} \mathrm{CD} 62 \mathrm{~L}^{-}\right)$in the spleen were detected and analyzed (Fig. 5E-G and Fig. S4). As expected, the levels of splenic $T_{E M}$ cells gradually increased between day 1 and day 7 in the best-performing groups (37 MBq 2-[ $\left[{ }^{18} \mathrm{~F}\right] \mathrm{FDG}+400 \mathrm{mg}$ aPD-L1 @ 4 h), which was higher than the saline group. Further, for 2-[ $\left.{ }^{18} \mathrm{~F}\right] \mathrm{FDG}$-induced immunotherapy, the splenic $\mathrm{T}_{\mathrm{EM}}$ cells remained at a high level until 60 days after the combined treatment. These results demonstrated that the prevention of tumor recurrence by T2T-based immunotherapy was credited to the activation of immunological memory effect.

\section{Coupling 2-[18 F]FDG with anti-PD-L1 antibody reprograms TIME}

The impact of radiotracer to PD-1/PD-L1 ICBs is multifaceted. Fig. 6A summarizes the potential mechanisms of T2T-based immunologic responses, which can help us reconsider the role of $2-\left[{ }^{18} \mathrm{~F}\right] \mathrm{FDG}$ in tumor imaging and immunotherapy. Transcriptomics analysis focused on the DEGs during the treatment process. In Fig. 6B, compared with the saline group, the CD274 gene (PD-L1) in the tumor of 2$\left[{ }^{18} \mathrm{~F}\right] \mathrm{FDG}$ or 2-[ $\left[{ }^{18} \mathrm{~F}\right] \mathrm{FDG}+\mathrm{aPD}-\mathrm{L} 1$ @ 4 h groups was upregulated on day 1. A few days later, this indicator showed a fairly noticeable decline.

As shown in Fig. S5, compared to the saline group and APD-L1 group, the enhanced change of tumor PDL1 level following 2- $\left[{ }^{18} \mathrm{~F}\right] \mathrm{FDG}$ alone or combined immunotherapy strongly predicted response to 
radionuclide stimulus. Then this indicator showed a tendency to decrease during the later period. Contrary to the trend of PD-L1, we observed increased level of IFN- $\gamma$ for radiotracer-induced immunotherapy (Fig. S6A,B). Further results indicated that both the CD $4^{+} \mathrm{Th} 1\left(\mathrm{IFN}-\mathrm{\gamma}^{+} \mathrm{CD} 4^{+} \mathrm{T}\right.$ cells) and CD8 ${ }^{+}$cytotoxic $T$ lymphocytes (IFN- $\gamma^{+} \mathrm{CD} 8^{+} \mathrm{CTLs}$ ) in TIME were enhanced from day 1 to day 7 in the group of 2-[18 F]FDG + aPD-L1 mAb @ 4 h, whereas the levels of these indicators were unaltered in the saline group. Meanwhile, tumor samples were harvested for detecting proliferation and apoptosis by immunofluorescence staining of Ki67 and Caspase3. As depicted in Fig. S6C, the dynamic change of PDL1 expression from day 1 to day 7 in the combination therapy groups were further validated. As expected, at the corresponding time points, the Ki67 indexes were significantly higher in saline groups. While the positive rate of Caspase 3 expression in the combination group was significantly higher than that in saline groups.

Similar to the IHC and immunofluorescence results, tumor PD-L1 expression measured by flow cytometry showed a decreasing trend during the period of day 1 to day 7 in groups containing radiotracer (Fig. 6C,D). ELISA assays were performed to measure the levels of immunostimulatory cytokines in the serum of mice. The combination of 2-[18 F]FDG and aPD-L1 mAb increased the production of IFN-y, TNF- $a$ and IL6 , and maintained for a long period in blood, which might also explain for the unexpected synergistic anticancer efficacy (described in Fig. 6E). Additionally, the flow cytometric results in Fig. S7A,B showed that intratumoral $\mathrm{CD} 4^{+} \mathrm{Th} 1$ and $\mathrm{CD} 8^{+} \mathrm{CTLs}$ become exhausted on day 3 and day 7 in the $2-\left[{ }^{18} \mathrm{~F}\right] \mathrm{FDG}$ group. However, the addition of aPD-L1 mAb @ 4 h significantly increased numbers of CD $4{ }^{+}$Th1 and $\mathrm{CD} 8^{+} \mathrm{CTLs}$ compared to the other groups. However, as one type of $\mathrm{CD} 4^{+} \mathrm{T}$ cells, the immunosuppressive $\mathrm{CD}^{+}{ }^{+} \mathrm{FOXP3}^{+}$regulatory T cell (Treg) in tumors showed a decrease in 2-[18 F]FDG + aPD-L1 mAb @ $4 \mathrm{~h}$ group. Specifically, further comparative analysis showed a significant increase of CD $4^{+}$Th1/Treg and $\mathrm{CD} 8^{+} \mathrm{CTLs} /$ Treg ratios in the combination group (Fig. 6F). In the $2-\left[{ }^{18} \mathrm{~F}\right] \mathrm{FDG}$ group, we observed slight increase in $\mathrm{CD} 4^{+}$Th1/Treg and $\mathrm{CD} 8^{+} \mathrm{CTLs} /$ Treg ratios on day 1 . Over time, these ratios seem to be on a downward trend.

Other alterations of immune cells are also notable. M2-like macrophages, M1-like macrophages, myeloidderived suppressor cells (MDSCs) and dendritic cells (DCs) were detected through flow cytometry (Fig. 6G and Fig. S8). The MC38 tumor-bearing mice that received 37 MBq 2-[ $\left[{ }^{18} \mathrm{~F}\right] \mathrm{FDG}+400 \mathrm{mg}$ aPD-L1 @ $4 \mathrm{~h}$ decreased the fraction of $\mathrm{M} 2$-like macrophages $\left(\mathrm{CD} 206^{+} \mathrm{CD} 11 \mathrm{~b}^{+} \mathrm{F} 4 / 80^{+}\right)$in the first few days, implying the reduced immunosuppression. While an opposite tendency was observed in the $2-\left[{ }^{18} \mathrm{~F}\right] \mathrm{FDG}$ group. Similarly, the radionuclide-induced PD-1/PD-L1 immunotherapy generated a 2-3 fold decrease in the fraction of MDSCs $\left(\mathrm{CD} 45^{+} \mathrm{CD} 11 \mathrm{~b}^{+} \mathrm{Gr}-1^{+}\right)$compared with the saline group or 2-[ $\left.{ }^{18} \mathrm{~F}\right] \mathrm{FDG}$ alone. We also detected pro-inflammatory M1-like macrophages (iNOS ${ }^{+} \mathrm{CD} 11 \mathrm{~b}^{+} \mathrm{F} 4 / 80^{+}$) and activated DCs $\left(\mathrm{CD} 80^{+} \mathrm{CD} 86^{+}\right)$in the TIME. Treatment with 2-[18 $\left.\mathrm{F}\right] \mathrm{FDG}+\mathrm{aPD}-\mathrm{L} 1$ @ $4 \mathrm{~h}$ led to a significant increase in the fraction of M1-like macrophages, further indicating the repolarization of M2-like macrophages or recruitment of M1-like macrophages. KEGG enrichment analysis was performed to identify the detailed immune activation associated pathways and inflammatory signaling pathways mediated by the 
therapeutic strategy of 2-[ $\left.{ }^{18} \mathrm{~F}\right] \mathrm{FDG}+$ aPD-L1 @ 4 h. Several representative pathways were shown in Fig. 7A. The most significant differences between the saline and 2-[18 F]FDG + aPD-L1 @ 4 h groups were found in the antigen processing and presentation, phagosome, cell adhesion molecules, the NOD-like receptor signaling pathway, cytokine-cytokine receptor interaction and Th17 cell differentiation. Moreover, we went a step further to confirm that the external radiotracers would affect the expression of PD-1, another key component of immune checkpoint blockade (Fig. 7B). Together, these profiles further confirmed that the radionuclide-induced PD-1/PD-L1 immunotherapy could inflame the TIME and activate the immune system.

Coincidentally, consistent with the aforementioned heat map of DEGs (Fig. 1G), subsequent therapeutic trials confirmed that MC38 tumor was more susceptible to $2-\left[{ }^{18} \mathrm{~F}\right] \mathrm{FDG}$ than CT26. We investigated the antitumor efficacy of 2-[ $\left.{ }^{18} \mathrm{~F}\right] \mathrm{FDG}$ plus aPD-L1 mAb in CT26 tumor, another widely used murine colorectal tumor model. Although to a less extent still significant when compared to the MC38 section, the growth of CT26 tumors was greatly suppressed in groups of $18.5 \mathrm{MBq}$ or $37 \mathrm{MBq} 2-\left[{ }^{18} \mathrm{~F}\right] \mathrm{FDG}+400 \mathrm{mg}$ aPD-L1 mAb @ 4 h, resulting in prolonged overall survivals (Fig. S9A-C). Dynamic changes of PD-L1 in tumor and cytokine levels in blood were found in the group of combining 2-[ $\left.{ }^{18} \mathrm{~F}\right] \mathrm{FDG}$ with aPD-L1 @ 4 h during the therapy period (Fig. S9D-F). Therapeutically, the activation of $\mathrm{CD} 4^{+}$and $\mathrm{CD} 8^{+} \mathrm{T}$ cells in the tumor and increased $\mathrm{CD}^{+}{ }^{+}$Th1/Treg and $\mathrm{CD} 8^{+} \mathrm{CTLs} /$ Treg levels highlighted the potential of the effective coordination to enhance antitumor immunity (Fig. S9G). Previous studies suggested that the less-immunogenic and microsatellite-instable CT26 model did not respond to irradiation with increased PD-L1 expression ${ }^{31,32}$. To some extent, this study describes a new method to overcome this setback via radionuclide-induced immunotherapy.

\section{Discussion}

ICB antibodies targeting the PD-1/PD-L1 pathway have revolutionized the oncology. Because patients with positive PD-L1 expression generally have better objective response rates (ORRs), practitioners have very high expectation for selecting eligible candidates for ICB therapy based on SPECT/PET imaging. However, to best realize such intention, it is important, as a first step, to find out whether the PD-L1 expression would be influenced by a T2T effect of the employed radiotracers (not just 2-[ $\left.{ }^{18} \mathrm{~F}\right] \mathrm{FDG}$, also other radiotracers in the immunoimaging toolbox). Based on our observations in this study, the dose- and time-dependent T2T effect directly influences the accuracy and rationality of radioimmunoimagingguided PD-L1 identification and therefore should not be overlooked. Fig. 6A summarizes the relationship between the radiotracer and multifaceted immunologic responses (including dynamic changes of CD $4^{+}$ Th1 and CD $8^{+}$CTLs, etc.), which can help us reconsider cautiously the role of radiotracers including 2$\left[{ }^{18} \mathrm{~F}\right] \mathrm{FDG}$ in tumor imaging and immunotherapy. In brief, the dynamic interferences and misleading recommendations from radiotracers need to be redefined. Unfortunately, to date, no data are available from clinical or preclinical trials to discuss this intractable question. In general, from our point of view, a multidimensional assessment based on biopsy specimens before radiotracer injection (initial TIME 
status) and radioimmunoimaging feedback (T2T-based immune response) should be emphatically considered prior to initiation of immunotherapy to guide clinicians in adjusting regimens of ICB therapy as appropriate.

Therapeutically, T2T responses in multiple tumor types observed in our experiments have spurred us to investigate the radionuclide-induced immunotherapeutical effects and made us fully aware that immune cells need certain amount of time to be activated and PD-L1 expression needs to be stimulated in tumor cells firstly ${ }^{31}$. Subsequent studies provide evidence on the necessity and timeliness of the participation of aPD-L1 mAb. Taking MC38 tumor model receiving 2-[ ${ }^{18}$ F]FDG plus aPD-L1 for example, of all the groups, the overall therapeutic outcomes of the 6 -h interval were most impressive. That is to say, an immunefavorable TME is activated by targeted radionuclide and therapeutic benefit is enhanced by aPD-L1 mAb subsequently. Meanwhile, it has been shown that upregulation of PD-L1 in the tumor can result in an increased demand for antibody, which seems to be a reasonable approach.

The radiotherapeutic isotopes $\left({ }^{177} \mathrm{Lu},{ }^{90} \mathrm{Y},{ }^{111} \mathrm{In},{ }^{131} \mathrm{I}\right.$, etc.), which generally have blast of radiation, are actively pursued in targeted radionuclide therapy to destroy the tumor cells. Whilst the therapeutic potential of positron-emitting agents is still underexplored. Ideally, based on the consideration of radionuclide characteristics, ${ }^{18} \mathrm{~F}$-based nuclear therapy will lead to a reduction in cost and radiation. Theoretically, 2-[18 $\mathrm{F}] \mathrm{FDG}$ emits positrons and should kill cancer cells in the same manner as electrons. In previous studies, high-dose 2-[18 F]FDG (111-222 MBq per mouse) was used for the radiomolecular therapy of cancer in mouse models and effective therapeutic response (incomplete cure) was observed ${ }^{33-}$ ${ }^{35}$. Meanwhile, radiotoxicity of $2-\left[{ }^{18} \mathrm{~F}\right] \mathrm{FDG}$ (222 MBq per mouse) was not found ${ }^{33}$. It is well known that radiation can enhance antitumor immunity by damaging DNA and inducing apoptosis/necrosis of the tumor cells. On this basis, with the synergy of aPD-L1 mAb and properly scheduled time window, it is possible to further drive ${ }^{18} \mathrm{~F}$ dosage lower, which could be favorable for clinical application. However, to date, the potential of immune-mediated response enhanced by ${ }^{18} \mathrm{~F}$ remains largely unexplored. Fortunately, on basis of our findings, we reason that the smart T2T immunoediting effect induced by 2$\left[{ }^{18} \mathrm{~F}\right] \mathrm{FDG}$ has the potential to boost immune activation in a manner that is synergistic with PD-1/PD-L1 ICB immunotherapy. In MC38 tumor model, the optimal dose of $2-\left[{ }^{18} \mathrm{~F}\right] \mathrm{FDG}$ is $37 \mathrm{MBq}$ per mouse, which is far below the physiological human maximum tolerated dose.

More interestingly, the relationship between radionuclides, PD-L1 upregulation and timeline could be drawn directly into a tumor-nuclide-time code (similar to Fig. 1C) and used to differentiate tumor types that are sensitive to T2T immunotherapy, which aligns well with precision cancer immunotherapy. Besides the radionuclide, dose, timing and sequencing setting discussed above, the target site of the probe is an important consideration for the design of the therapy project. Of note is that targeting PD-L1 on tumor cells is not the only choice to pave the way for anticancer-T2T-based ICB immunotherapy. The other ICB targets, such as CTLA-4, STING, VISTA etc., may also be used in the radiotracers combined immunotherapy schema. Various radiolabeled specific targeting agents (FDG, RGD, fibroblast-activating protein (FAP), prostate-specific membrane antigen (PSMA), octreotate (TATE), folic acid (FA), bombesin 
(BBN), etc.), nanoprobes, albumin binders and biomacromolecule can be used to enrich the T2T toolbox. And the application scenarios could even be extended to allow the multi-targets or multi-isotopes strategy

if necessary. Compared with radiotherapy nuclides, such as ${ }^{177} \mathrm{Lu}$ and ${ }^{131} \mathrm{I}$, diagnostic radiotracers as immunomodulators have shorter half-life, quicker in vivo clearance rate and higher biological safety (without $\beta^{-}$radiation), which could be widely used in the patients. All these smart T2T strategies will unquestionably contribute to the vision of precision cancer immunotherapy. Compared with the traditional local radiotherapy only for the irradiated area (within the irradiated field), the addition of appropriate radiotracer and inhibitor seems to be a more attractive option with "cross-fire" effect, which can augment the depth and duration of responses, boost the systemic antitumor immune response, especially for multiple lesions, distant metastases and tiny foci. Such a breakthrough will clinically put the nuclear medical diagnosis and ICBs together in a series, and thus truly build an image-guided platform for tumor theranostics.

Studies have shown that one of the main challenges for ICB immunotherapy lies in "cold" tumor with limited T-cell responses ${ }^{36,37}$. Fortunately, as summarized in Fig. 6F, we found that radionuclide-based ICB immunotherapy synergistically enhanced antitumor immunity by promoting critical parameters of $\mathrm{CD} 4^{+}$ Th1/Treg and CD8 ${ }^{+}$CTLs/Treg ratios in TIME. As we know, CD4 and CD8-positive T-cells are important hallmarks of transition from "cold" TIME to "hot", which is critical to realize ideal ICB outcomes. And Tregs are defined as immunosuppressors for maintaining immunological tolerance, which is associated with a poor prognosis. Optimistically, this smart T2T effect seems to be an attractive option to eliminate treatment resistance of patients with low or no PD-L1 expression in pretreatment tumors and improve clinical outcomes.

In summary, we have demonstrated that imaging radionuclides induce significant PD-L1 upregulation in tumor cells. From a predictive perspective, it is necessary to develop reliable imaging strategies for precisely monitoring PD-L1 expression. Besides, further studies are also underway to take corrective actions for improving the accuracy of immuno-PET/SPECT in guiding PD-L1 expression in patients. Furthermore, this work provides insight into the new role of imaging tracer such as $2-\left[{ }^{18} \mathrm{~F}\right] \mathrm{FDG}$ as an immunomodulator. Understandably, these results highlight the T2T effect for a new paradigm in tumor theranostics and provide practical guidance for precision cancer immunotherapy.

\section{Online Methods}

\section{General Remarks}

All chemicals were obtained commercially. The murine colorectal cancer cell lines (MC38 and CT26) were purchased from the China National Infrastructure of Cell Line Resource. InvivoPlus anti-mouse PD-L1 mAb (BP0101) was purchased from BioCell Co. Ltd. Anti-human PD-L1 (SHR-1316) was provided from Hengrui Co. Ltd., China. Antibodies used for western blot assays were purchased from Abcam or Cell signaling Technology Inc. (USA). PET imaging studies were performed by a microPET/CT device (Siemens Inveon PET/CT, USA). The radioactivity was measured with y-counter (Wizard 2480, Perkin- 
Elmer, USA) and CRC-25R dose calibrators (CAPIN-TEC Inc. USA). Cell immunofluorescence was performed by laser scanning confocal microscope (Olympus FV1200, Japan). IHC of tissues were detected by microscope of Leica DM4 B (Leica, German). Flow cytometry was detected with Beckman coulter CytoFLEX (Beckman coulter, USA).

\section{Animal Models}

$\mathrm{BALB} / \mathrm{c}$ mice, BALB/c nude mice and C57BL/ 6 mice (female, $6-8$ weeks age, $16-18 \mathrm{~g}$ body weight) were used in our studies. All animals were randomly assigned to the experimental groups, obtained from Vital River Laboratory Animal Technology Co., Ltd. (Beijing, China) and housed with a $12 \mathrm{~h}$ light-dark cycle at $22{ }^{\circ} \mathrm{C}$ and food and water ad libitum. All animal protocols were approved by the Institutional Animal Care and Use Committee of National Center for Xiamen University (ID XMULAC20190150).

Murine subcutaneous tumor models: The right rear flanks of female BALB/c mice were given a suspension of CT26 colorectal tumor cells $\left(2 \times 10^{6}\right.$ tumor cells in $100 \mu \mathrm{L}$ PBS) subcutaneously. For female C57BL/ 6 mice, subcutaneous tumors were generated by inoculating MC38 colorectal cancer cells $\left(2 \times 10^{6}\right.$ tumor cells in $\left.100 \mu \mathrm{L} \mathrm{PBS}\right)$ suspension. The tumor volume $\left(\mathrm{mm}^{3}\right)$ was using a digital vernier caliper and calculated as length $\times$ width $^{2} / 2$.

NSCLC PDX models: In this study, fresh tumor samples from NSCLC patients were implanted subcutaneously into BALB/c nude mice to establish NSCLC-PDX models, which retain the morphology and molecular signatures of the corresponding parental tumor. The studies involving human participants were approved by the Clinical Research Ethics Committee of the First Affiliated Hospital of Xiamen University (ID KYZ2017-001). The informed consent was obtained from patients prior to the utilization of all clinical samples and data. Briefly, NSCLC specimens were surgically removed and immediately placed in DMEM supplemented with $2 \%$ antibiotics. To establish PDX models, the right upper limbs of female BALB/c immune-deficient mice were implanted subcutaneously fresh tumor specimens within an average of two hours following the patient's surgery. The mouse was euthanized to remove the tumor until it reached a volume of $1000 \mathrm{~mm}^{3}$. For investigational purposes, the tumor fragments were implanted in $\mathrm{BALB} / \mathrm{c}$ nude mice to expand a higher number of PDXs to obtain statistically relevant results. Models were subjected to imaging study when the tumor volume reached $100 \mathrm{~mm}^{3}$.

\section{Synthesis of $\mathrm{Cy}_{5.5^{-}-\mathrm{aPD}-\mathrm{L} 1}$}

Cy5.5-NHS (50 $\mu$ g dissolved in $10 \mu \mathrm{L}$ DMSO) was added into anti-human aPD-L1 mAb in PBS and shocked at RT for $2 \mathrm{~h}$ to get $\mathrm{Cy}_{5.5}$-aPD-L1. And then, free Cy5.5-NHS was removed by ultrafiltration centrifuge tubes with an MWCO value of $3 \mathrm{kDa}$.

\section{Cellular uptake of $\mathrm{Cy}_{5.5^{-}} \mathrm{aPD}-\mathrm{L} 1$}

For flow cytometry experiments, the cells were incubated with 2-[18 $\mathrm{F}] \mathrm{FDG}(1.85 \mathrm{MBq} / \mathrm{mL})$ for overnight and then replaced with fresh medium (without serum) containing $\mathrm{Cy}_{5.5}$-aPD-L1. After further incubation 
for different time points $(10,30,60,120,240 \mathrm{~min})$ at $37^{\circ} \mathrm{C}$, the cells were washed two times with cold PBS and collected, then re-suspended in $200 \mu \mathrm{L}$ PBS for flow cytometric analysis (BD Biosciences). ${ }^{18} \mathrm{~F}$ untreated cells were used as control. A total of 10,000 events were collected for each sample.

\section{RNA Isolation, First Strand cDNA Synthesis and RT-qPCR}

To quantify PD-L1 mRNA expression, CT26 and MC38 colorectal tumor cells were seeded in 6-well plates to grow overnight. Then the cells were incubated with radionuclide $\left(1.85 \mathrm{MBq} / \mathrm{mL}\right.$ of ${ }^{18} \mathrm{~F},{ }^{99 \mathrm{~m}} \mathrm{Tc}$, ${ }^{177} \mathrm{Lu}$ or ${ }^{64} \mathrm{Cu}$ ) for different time $(2 \mathrm{~h}, 4 \mathrm{~h}, 24 \mathrm{~h})$ at $37{ }^{\circ} \mathrm{C}$. Total RNA was isolated from CT26 and MC38 tumor cells using the RNeasy Mini Plus Kit (Sangon biological). The cDNA was obtained by reverse transcription using the Maxima First Strand cDNA Synthesis Kit for RT-qPCR (Thermo Fisher Scientific), according to the manufacturer's instructions. iScript and SsoAdvanced SYBR Green supermix (BioRad) were used for RT-PCR gene expression analysis on ABI StepOne Plus Real-Time PCR system. Each reaction mixture contained $10 \mu \mathrm{L}$ of SybrGreen qPCR Master Mix, $0.4 \mu \mathrm{L}$ of forward and reverse primers (Sangon biological), and $2 \mu \mathrm{L}$ of cDNA. The qPCR conditions were: $50^{\circ} \mathrm{C}$ for $3 \mathrm{~min}, 95^{\circ} \mathrm{C}$ for $3 \mathrm{~min}$, followed by 45 cycles at $95{ }^{\circ} \mathrm{C}$ for $5 \mathrm{~s}$, and $60^{\circ} \mathrm{C}$ for $30 \mathrm{~s}$. Amplification of specific transcripts was confirmed by melting curve profiles generated at the end of the PCR program. Expression levels of target genes were normalized to the housekeeping gene ( $\beta$-actin) and were calculated based on the comparative cycle threshold method $\left(2^{-\Delta \Delta \mathrm{Ct}}\right)$ and shown by heat map with Graphpad prism 7.0 software. All the measurements were performed in triplicate. Primer sets used were as follows:

PD-L1-F: CCTGCTTGCGTTAGTGGTGT;

PD-L1-R: GCGTGATTCGCTTGTAGTCC;

ß-actin-F: GTGCTATGTTGCTCTAGACTTCG;

ß-actin-R: ATGCCACAGGATTCCATACC.

\section{Transcriptomics Study}

Transcriptomics study was performed in HaploX Genomics Center, Ltd.

MC38 and CT26 tumor cells were seeded in 6-well plates overnight and then incubated with 2- $\left[{ }^{18} \mathrm{~F}\right] \mathrm{FDG}$ $(1.85 \mathrm{MBq})$ for $24 \mathrm{~h}$. After that, these tumor cells were collected and the high-throughput sequencing was performed. $2-\left[{ }^{18} \mathrm{~F}\right] \mathrm{FDG}$-untreated cells were used as control.

MC38 tumor tissue samples of different groups were collected for high-throughput sequencing. Reference genome and gene model annotation files were downloaded from the genome website directly. Paired-end clean reads were aligned to the reference genome using HISAT2 v2.1.0 (hierarchical indexing for spliced alignment of transcripts), which is a highly efficient system for aligning reads from RNA sequencing experiments. 


\section{Immunofluorescent, Histology, Immunohistochemistry and Microscopy}

For immunofluorescent analysis of singe-cell, CT26, MC38, 4T1 and B16F10 tumor cells were seeded in confocal dishes. Each sample was fixed with $100 \mu \mathrm{L}$ of $4 \%$ paraformaldehyde for 10 minutes. After that, the cells were washed three times with PBS and incubated with $10 \%$ goat serum for 30 min to reduce nonspecific binding. Cells were stained with the first antibody PD-L1 (primary antibody, Abcam, USA) overnight, rinsed three times with PBS. After cells were stained for $1 \mathrm{~h}$ with secondary antibody Alexa Fluor ${ }^{\circledR}$ Plus 488-conjugated IgG and washed three times with PBS, cell nuclei were stained blue with DAPI (Invitrogen Molecular Probes, USA). For histological analysis, tissue specimens were fixed with 10\% buffered formalin, dehydrated in ethanol, embedded with paraffin and stained with H\&E.

Immunohistochemistry on frozen or paraffin-embedded mouse tissues was performed using antibodies directed against PD-L1, CD4, IFN-ץ, CD8. For paraffin-embedded samples (PD-L1, CD4, IFN- $\gamma$, CD8), samples were dewaxed in ethanol, followed by antigen retrieval with $0.01-\mathrm{M}$ sodium citrate with $0.05 \%$ Tween. Immunofluorescence staining on frozen mouse tissues was performed using antibodies against PD-L1, ki67, caspase3 and DAPI. Immunofluorescence images were acquired using the Zeiss LSM880 confocal microscope with ZEN 2010 software. Histological and Immunohistochemistry images were acquired using the Leica DM4 B upright digital research microscopes (Leica, Germany) with Leica Application Suite X (LAS X). All the images were quantitatively analyzed with Image J 7.0 software and data were shown by a histogram with Graphpad prism 7.0 software.

\section{Western Blot Analysis}

Western Blot was performed as described previously ${ }^{38}$, with minor modifications. Briefly, cell lysates were made in ice-cold RIPA buffer containing complete protease inhibitor cocktail and phosphatase inhibitor cocktail (Sigma). Total protein was quantified using the BCA Assay according to the manufacturer's instructions (ThermoFisher). 10\% Bis-Tris polyacrylamide gels were equiloaded with $20 \mu \mathrm{g}$ of protein, electrophoresed at $120 \mathrm{~V}$, and electro-transferred to PVDF membranes. After blocking with 5\% BSA, membranes were probed with primary antibodies to PD-L1, NF-KB p65, Phospho-NF-KB p65, IRF3, Phospho-IRF-3 and $\beta$-actin. Blots were developed by ECL (Thermo Fisher Scientific).

\section{Flow Cytometry of Cell Lines and Organs}

Tumor cells were seeded in 6-well plates overnight and treated with different radionuclides $\left({ }^{18} \mathrm{~F}\right.$, ${ }^{99 \mathrm{~m}} \mathrm{Tc}$, $\left.{ }^{177} \mathrm{Lu},{ }^{64} \mathrm{Cu},{ }^{131} \mathrm{l}\right)$. Saline-treated cells were used as control group. After incubation with different time (0.5 h, $2 \mathrm{~h}, 4 \mathrm{~h}, 8 \mathrm{~h}$ and $24 \mathrm{~h}$ ), cells were harvested and washed with cold PBS, then stained with mouse antiPD-L1 mAb (MIH6) (Abcam, USA). The second antibody labelled with fluorescein isothiocyanate (FITC) was used for detecting first antibodies. Fluorescence intensities of the stained cells were analyzed using a FACS Aria III flow cytometer (BD Biosciences). Data were assessed and analyzed quantitatively by FlowJo software version 10 (FlowJo). 
Single-cell suspensions of mouse tumors were prepared for flow cytometry as described previously ${ }^{39}$. In brief, samples were harvested and cut into small fragments $\left(1-2 \mathrm{~mm}^{3}\right)$, and placed in DMEM containing Collagenase IV ( $1 \mathrm{mg} / \mathrm{mL}$; Gibco, USA), trypsin inhibitor ( $1 \mathrm{mg} / \mathrm{mL}$; EMD Millipore), and DNase I ( $2 \mathrm{U} / \mathrm{mL}$; Promega). The fragments were then incubated at $37^{\circ} \mathrm{C}$ for $60 \mathrm{~min}$ with gentle shaking every $10 \mathrm{~min}$. Specimens were passed through a $70 \mu \mathrm{m}$ mesh and centrifuged at $350 \mathrm{~g}$ for $5 \mathrm{~min}$. Red blood cells were eliminated from the samples with a hypo-osmotic red blood cell lysis buffer (Solarbio). Each sample was fixed with $100 \mu \mathrm{L}$ of $4 \%$ paraformaldehyde for 10 minutes. After that, the cells were collected and washed three times with PBS and incubated with $10 \%$ goat serum to reduce nonspecific binding. For detecting PD-L1 expression and T cell alteration, preprocessed cells were stained with the first antibodies (anti-PDL1, CD4 / IFN-ץ / Foxp3, CD8 / IFN-ץ), second antibodies (rat, rabbit, or mouse anti-mouse) labelled with Alexa Fluor ${ }^{\circledR}$ Plus 488, Cyanine3 or Alexa Fluor ${ }^{\circledR}$ Plus 647 (Invitrogen) were used for detection first antibodies, respectively. In addition, other immune cells were defined as follows: M1 macrophage: iNOS ${ }^{+}$, $\mathrm{CD}_{11} \mathrm{~b}^{+}, \mathrm{F} 4 / 80^{+}$; $\mathrm{M} 2$ macrophage: $\mathrm{CD} 206^{+}, \mathrm{CD}_{11} \mathrm{~b}^{+}, \mathrm{F} 4 / 80^{+}$; dendritic cell (DC): $\mathrm{CD} 80^{+}, \mathrm{CD}^{+} 6^{+}$; myeloidderived suppressor cells (MDSC): $\mathrm{CD} 45^{+}, \mathrm{CD} 11 \mathrm{~b}^{+}, \mathrm{Gr}-1^{+}$, respectively. Effector memory T cells of spleen were defined as follows: $\mathrm{CD} 44^{+}, \mathrm{CD}^{+}, \mathrm{CD} 62 \mathrm{~L}^{-}$or $\mathrm{CD} 44^{+}, \mathrm{CD} 8^{+}, \mathrm{CD} 62 \mathrm{~L}^{-}$. PDX model tumors were dissected and stained with anti-human PD-L1 mAb [EPR19759] to examine PD-L1 expression change.

\section{Cytokine Analysis}

Serum samples were isolated from mice after various treatments and diluted for analysis. The proinflammatory cytokines including TNF-a, IFN- $y$ and IL- 6 were determined by using enzyme-linked immune sorbent assay (ELISA) kits according to vendors' protocols (Dakewe biotech). The data were calculated and shown in a bar chart with Graphpad prism 7.0 software.

\section{In Vivo Anticancer Efficacy}

As the tumor volume reached about $50 \mathrm{~mm}^{3}$, the CT26 or MC38 tumor-bearing mice were randomly divided into different groups ( $\mathrm{n}=8$ per group) and treated with different schemes. An additional therapeutic course was scheduled on day 4. After initiation of radiotracer-related therapy, the feeding surroundings were shielded with lead bricks to protect them from any contact with extrinsic radiation. The tumor volume and body weight were monitored at the given time points. Mice were euthanasia if the tumor volume exceeded $1500 \mathrm{~mm}^{3}$. The percent survival of mice in each group was measured until all the mice had been sacrificed.

\section{Small Animal PET Imaging}

Small animal PET imaging studies were performed at the given time points under the approved guidelines. The injected doses were identical to that in therapeutic trials. During the scan procedure, anaesthesia was induced with isoflurane/air mixture to maintain spontaneous breathing of mice. Wholebody microPET imaging was carried out in tumor-bearing mice injected with 2-[ $\left.{ }^{18} \mathrm{~F}\right] \mathrm{FDG}$. Images were acquired directly following the acquisition of the CT. PET images were reconstructed using 2D/3D 
ordered-subset expectation-maximization (2D/3D OSEM) algorithm and with a Maximum a Posteriori Method (MAP). Injected dose and body weight were input before imaging to accomplish normalized and decay corrected radioactivity concentration. For quantitative comparisons, the tissue uptake was acquired by selecting the ROls on images.

\section{Statistical Analysis}

Statistical analyses were performed using an unpaired two-tailed Student's t-test with GraphPad Prism 7.0 software (GraphPad Software Inc.). Survival curve data were analyzed with the Kaplan-Meier method followed by the log-rank test with GraphPad Prism 7.0 software. Data are presented as the mean \pm standard deviation. Statistical significance is defined at the $* x \leq 0.05$ level.

\section{Reporting summary}

Further information on experimental design is available in the Nature Research Reporting Summary linked to this article.

\section{Data availability}

The authors declare that the data supporting the findings of this study are available within the article and its Supplementary Information Files or from the corresponding author on reasonable request.

\section{Declarations}

\section{Acknowledgments}

We thank Mingyan Xu, Xuanqin Wu, Qiannan Tong, Shifu Chen and Chao Lu at HaploX Biotechnology for technical assistance with transcriptome and signal-net analysis, and thank all members of the Center for Molecular Imaging and Translational Medicine for fruitful discussions. This study was financially supported by the National Natural Science Foundation of China (81901805, 21976150), Major Research Plan of the National Natural Science Foundation of China (91959122), Joint Fund of the National Natural Science Foundation of China - China National Nuclear Corporation for Nuclear Technology Innovation (U1967222), China Postdoc Innovation Talent Supporting Program (BX2017001420), Postdoctoral Science Foundation of China (2018M630732), and the National University of Singapore start-up fund (R180-000-017-133, R-180-000-017-733, and R-180-000-017-731).

\section{Author contributions}


Conception of the study and manuscript preparation: X.Z. and Z.G. Acquisition and analysis of the data: Z.G. and X.W. Histopathological characterization: C.S. and L.Y. Material support: H.C., Q.L., L.Z., J.H., and Y.L. Animal model support: X.Z. and L.F. Critical revision for important intellectual content: R.Z. and X.C.

\section{Competing interests}

The authors declare no competing interests.

\section{Additional information}

Supplementary information is available for this paper.

\section{References}

1. Sanmamed, M. F. \& Chen, L. A paradigm shift in cancer immunotherapy: from enhancement to normalization. Cel/ 175, 313-326 (2018).

2. Topalian, S. L., Taube, J. M. \& Pardoll, D. M. Neoadjuvant checkpoint blockade for cancer immunotherapy. Science 367, eaax0182 (2020).

3. Kluger, H. M. et al. PD-L1 studies across tumor types, its differential expression and predictive value in patients treated with immune checkpoint inhibitors. Clin. Cancer Res. 23, 4270-4279 (2017).

4. Zhou, Z. et al. PD-L1 expression is a predictive biomarker for CIK cell-based immunotherapy in postoperative patients with breast cancer. J. Immunother. Cancer 7, 228-228 (2019).

5. Zou, W., Wolchok, J. D. \& Chen, L. PD-L1 (B7-H1) and PD-1 pathway blockade for cancer therapy: Mechanisms, response biomarkers, and combinations. Sci. Transl. Med. 8, 328rv4 (2016).

6. Krieg, C. et al. High-dimensional single-cell analysis predicts response to anti-PD-1 immunotherapy. Nat. Med. 24, 144-153 (2018).

7. Finotello, F. et al. Next-generation computational tools for interrogating cancer immunity. Nat. Rev. Genet. 20, 724-746 (2019).

8. Mu, W. et al. Radiomics of ${ }^{18}$ F-FDG PET/CT images predicts clinical benefit of advanced NSCLC patients to checkpoint blockade immunotherapy. Eur. J. Nucl. Med. Mol. Imaging 47, 1168-1182 (2020).

9. Schwenck, J. et al. Cancer immunotherapy is accompanied by distinct metabolic patterns in primary and secondary lymphoid organs observed by non-invasive in vivo ${ }^{18}$ F-FDG-PET. Theranostics10, 925937 (2020).

10. Zhao, L. et al. Usefulness of $\left[{ }^{18} \mathrm{~F}\right]$ fluorodeoxyglucose PET/CT for evaluating the PD-L1 status in nasopharyngeal carcinoma. Eur. J. Nucl. Med. Mol. Imaging47, 1065-1074 (2020). 
11. 11. Bensch, et al. ${ }^{89} \mathrm{Zr}$-atezolizumab imaging as a non-invasive approach to assess clinical response to PD-L1 blockade in cancer. Nat. Med. 24, 1852-1858 (2018).

12. Niemeijer, A. N. et al. Whole body PD-1 and PD-L1 positron emission tomography in patients with non-small-cell lung cancer. Nat Commun. 9, 4664-4664 (2018).

13. Iravani, A. \& Hicks, R. J. Imaging the cancer immune environment and its response to pharmacologic intervention, part 1: the role of ${ }^{18}$ F-FDG PET/CT. J. Nucl. Med. 61, 943-950 (2020).

14. 14. Wei, W. et al. ImmunoPET: concept, design, and applications. Rev. 120, 3787-3851 (2020).

15. Mayer, A. T. \& Gambhir, S. S. The immunoimaging toolbox. J. Nucl. Med. 59, 1174-1182 (2018).

16. Iravani, A. \& Hicks, R. J. Imaging the cancer immune environment and its response to pharmacologic intervention, part 2: the role of novel PET agents. J. Nucl. Med. 61, 1553-1559 (2020).

17. He, C. et al. Core-shell nanoscale coordination polymers combine chemotherapy and photodynamic therapy to potentiate checkpoint blockade cancer immunotherapy. Nat. Commun. 7, 12499-12499 (2016).

18. Deng, L. et al. Irradiation and anti-PD-L1 treatment synergistically promote antitumour immunity in mice. J. Clin. Invest. 124, 687-695 (2014).

19. Bouillez, A. et al. MUC1-C integrates PD-L1 induction with repression of immune effectors in nonsmall-cell lung cancer. Oncogene 36, 4037-4046 (2017).

20. Xu, D. et al. PD-L1 expression is regulated by NF-KB during EMT signaling in gastric carcinoma. Onco Targets Ther. 12, 10099-10105 (2019).

21. Peng, J. et al. Chemotherapy induces programmed cell death-ligand 1 overexpression via the nuclear factor-kB to foster an immunosuppressive tumor microenvironment in ovarian cancer. Cancer Res. 75, 5034-5045 (2015).

22. Yamamoto, Y. \& Gaynor, R. B. IkappaB kinases: key regulators of the NF-kappaB pathway. Trends Biochem. Sci. 29, $72-79$ (2004).

23. Tanaka, Y. \& Chen, Z. J. STING specifies IRF3 phosphorylation by TBK1 in the cytosolic DNA signaling pathway. Sci. Signa/ 5, ra20 (2012).

24. Keestra-Gounder, A. M. et al. NOD1 and NOD2 signalling links ER stress with inflammation. Nature 532:394-397 (2016).

25. Spiotto, M., Fu, Y. X. \& Weichselbaum, R. R. The intersection of radiotherapy and immunotherapy: mechanisms and clinical implications. Sci Immunol. 1, EAAG1266 (2016).

26. Webb, L. V., Ley, S. C. \& Seddon, B. TNF activation of NF-KB is essential for development of singlepositive thymocytes. J Exp Med. 213, 1399-407 (2016).

27. Fehrenbacher, L. et al. POPLAR Study Group, Atezolizumab versus docetaxel for patients with previously treated non-small-cell lung cancer (POPLAR): a multicentre, open-label, phase 2 randomised controlled trial. Lancet 387, 1837-1846 (2016).

28. Gong, X. et al. Combined radiotherapy and anti-PD-L1 antibody synergistically enhances antitumour effect in non-small cell lung cancer. J. Thorac. Oncol. 12, 1085-1097 (2017). 
29. Mok, T. et al. Pembrolizumab versus chemotherapy for previously untreated, PD-L1-expressing, locally advanced or metastatic non-small-cell lung cancer (KEYNOTE-042): a randomised, open-label, controlled, phase 3 trial. Lancet 393, 1819-1830 (2019).

30. Rizvi, N. A. et al. Cancer immunology. Mutational landscape determines sensitivity to PD-1 blockade in non-small cell lung cancer. Science 348, 124-128 (2015).

31. 31. Derer, A. et al. Chemoradiation increases PD-L1 expression in certain melanoma and glioblastoma cells. Immunol. 7, 610-610 (2016).

32. Efremova, M. et al. Targeting immune checkpoints potentiates immunoediting and changes the dynamics of tumor evolution. Nat. Commun. 9, 32 (2018).

33. Fang, S. et al. Experimental study on the therapeutic effect of positron emission tomography agent $\left[{ }^{18} \mathrm{~F}\right]$-labeled 2-deoxy-2-fluoro-D-glucose in a colon cancer mouse model. Cancer Biother. Radiopharm. 25, 733-740 (2010).

34. Moadel, R. M. et al. Positherapy: Targeted nuclear therapy of breast cancer with 18F-2-deoxy-2-fluoroD-glucose. Cancer Res. 65, 698-702 (2005).

35. Moadel, R. M. et al. Positron emission tomography agent ${ }^{18} \mathrm{~F}$-2-deoxy-2-fluoro-d-glucose has a therapeutic potential in breast cancer. Breast Cancer Res. 5, 199-205 (2003).

36. Huang, L. et al. Mild photothermal therapy potentiates anti-PD-L1 treatment for immunologically cold tumors via an all-in-one and all-in-control strategy. Nat. Commun. 10, 4871-4871 (2019).

37. Galon, J. \& Bruni, D. Approaches to treat immune hot, altered and cold tumours with combination immunotherapies. Nat. Rev. Drug Discov. 18, 197-218 (2019).

38. Liu, Y. et al. TP53 loss creates therapeutic vulnerability in colorectal cancer. Nature 520, 697-701 (2015).

39. Seifert, L. et al. The necrosome promotes pancreatic oncogenesis via CXCL1 and Mincle-induced immune suppression. Nature 532, 245-249 (2016).

\section{Figures}



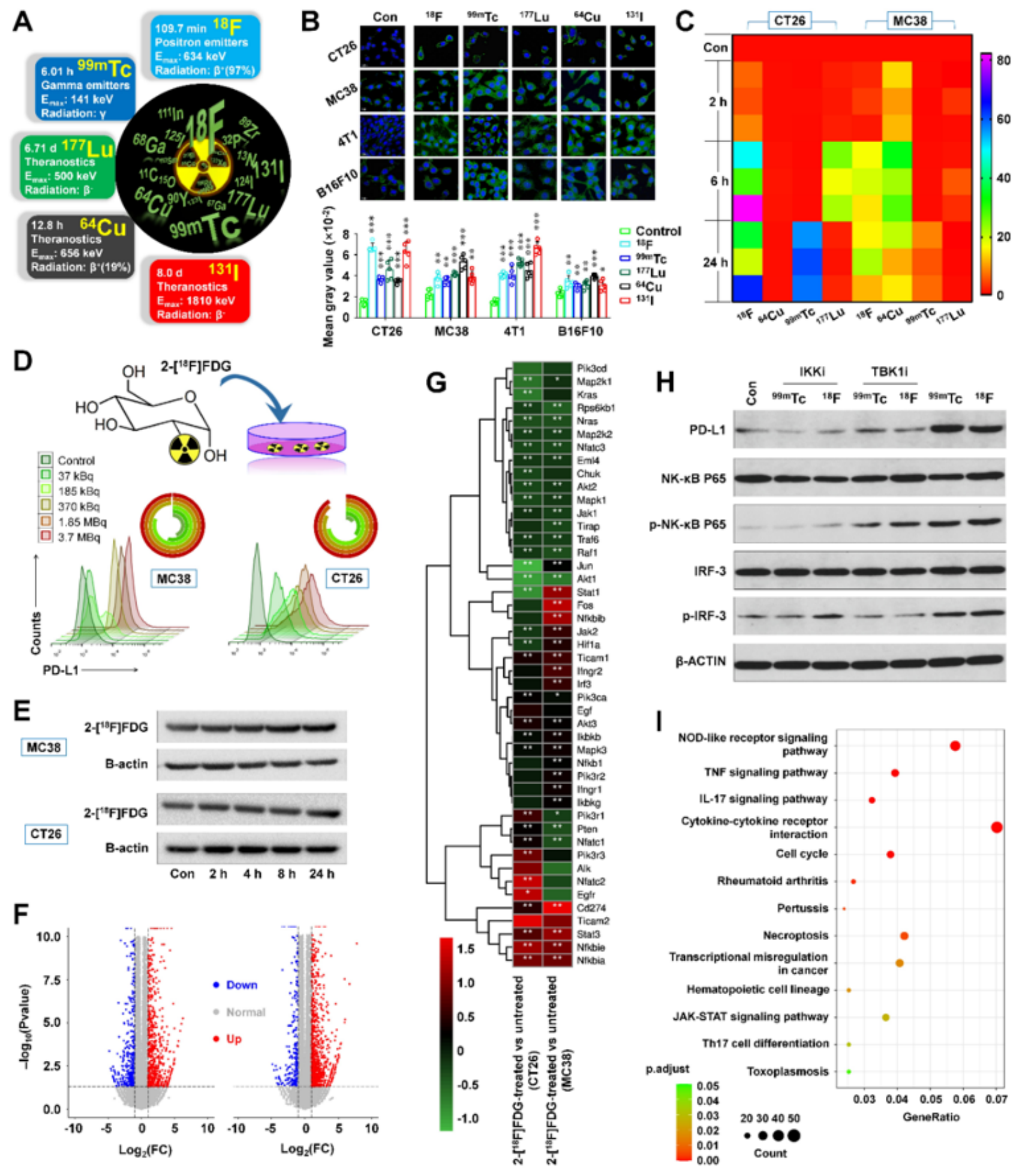

\section{Figure 1}

PD-L1 expression of tumor cell was significantly upregulated after stimulation with radionuclides. (A) Information of the radionuclides used in this research. (B) Confocal images of PD-L1 immunofluorescence staining in multiple tumor cell lines at $24 \mathrm{~h}$ after radionuclide irradiation (370 kBq). Representative histograms were used to present the upregulation of PD-L1 after radionuclide stimulation. Data are expressed as mean $\pm S D(n=3)$. Each experimental group was compared to the control $\left({ }^{\star} p \leq\right.$ 
$0.05 ;{ }^{* \star} p \leq 0.01 ;{ }^{* \star} p \leq 0.001$ ). (C) Heat map generated from quantitative RT-PCR was used to analyze the mRNAs encoding PD-L1 in MC38 and CT26 tumor cells after irradiation with different radionuclides. Untreated cells were used as controls. (D) The increase in PD-L1 expression on MC38 and CT26 tumor cells after irradiation with different doses of 2-[18F]FDG was quantified by flow cytometry. (E) The expressions of PD-L1 in MC38 (top) and CT26 (bottom) tumor cell lines after stimulation with 2-[18F]FDG $(1.85 \mathrm{MBq} / \mathrm{mL}$ ) were evaluated by Western blot. (F) Volcano plots of DEGs with MC38 (left) and CT26 (right) tumor cells at $24 \mathrm{~h}$ after irradiation with 2-[18F]FDG. (G) Heat map of DEGs in tumor cells $24 \mathrm{~h}$ after co-incubation with 2-[18F]FDG. The intensity of the color represents log2 fold-change (2-[18F]FDGtreated vs. untreated cells). Some up-regulated genes (e.g. Fos, Stat) are particularly associated with PDL1. (H) Radionuclides may up-regulate PD-L1 via the IRF-3 and NF-KB pathways. (I) KEGG bubble map of the functional pathways involved in the biological effect of MC38 tumor cells induced by 2-[18F]FDG. The sizes of dots represent the counts of DEGs in the corresponding pathway. 
A
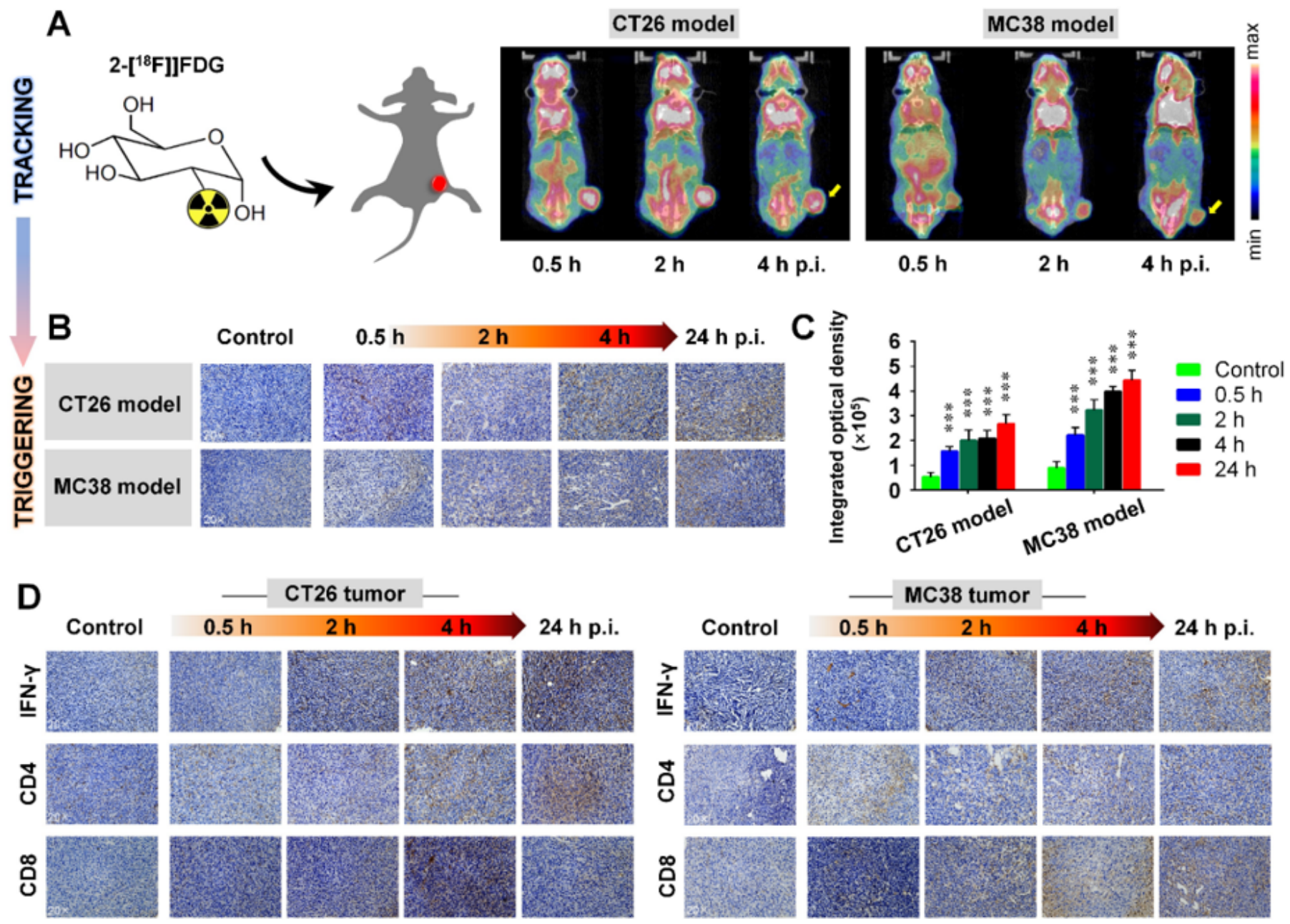

\section{CT26 tumor}

24 h p.i.
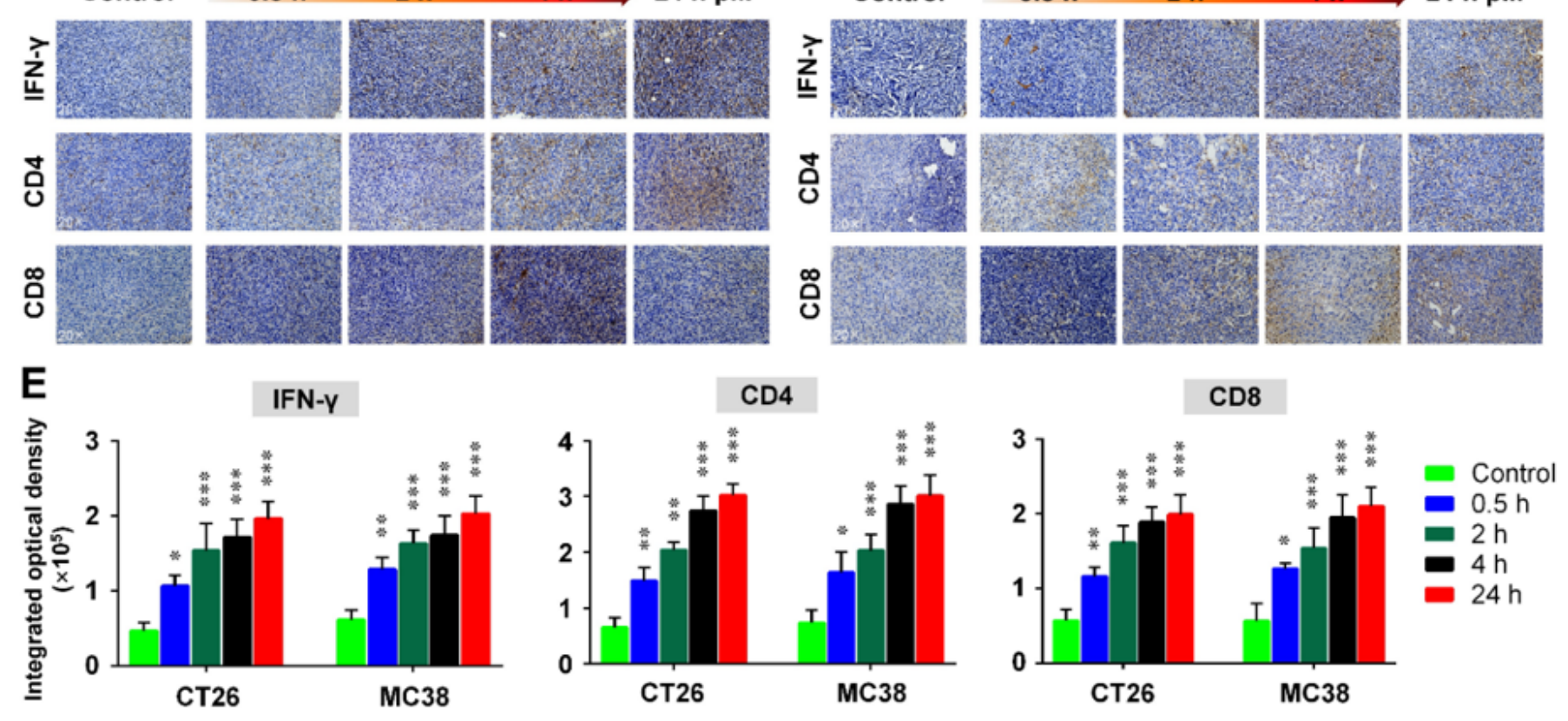

24 h p.i.
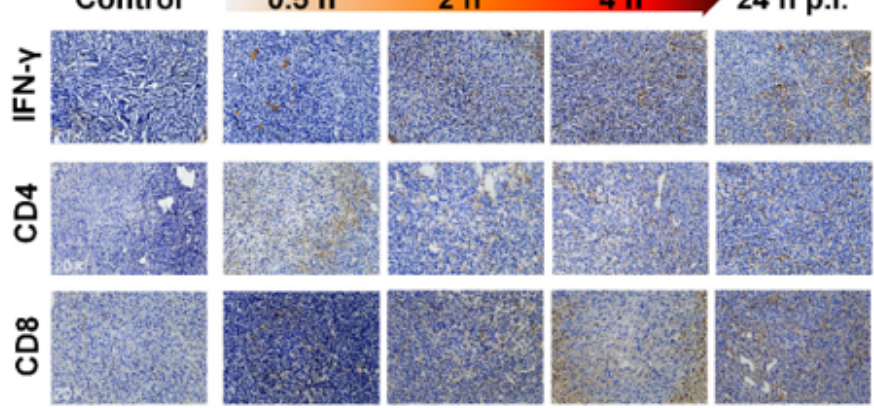

Figure 2

T2T immunoediting effect of the employed 2-[18F]FDG. (A) Coronal PET-CT images of 2-[18F]FDG in tumor-bearing mice (CT26 and MC38 tumor) at different time points. Tumor areas were indicated by yellow arrows. Images were adjusted to the same maximum value to show the clearance of 2-[18F]FDG. (B) IHC was performed to determine the PD-L1 expression on different tumor biopsies from CT26 and MC38 tumor models after injection of 2-[18F]FDG. Each tumor-bearing mouse was injected with 18.5 $\mathrm{MBq}$ of radiotracer via the tail vein. Afterward, tumors were harvested at $0.5,2,4$ and $24 \mathrm{~h}$ p.i. ( $\mathrm{n}=3$ per time point). The tumor biopsies of tumor mice without radiotracer injection were served as controls. (C) Quantification of IHC for PD-L1 expression via image J 7.0. (D) Representative IHC of tumor tissues for 
IFN- $\gamma+$, CD4+ and CD8+ T cells. The tumor samples of tumor-bearing mice without 2-[18F]FDG injection were served as control. (E) Quantification of IHC via image J 7.0 showed that 2-[18F]FDG could induce upregulation of pro-inflammatory cytokine IFN- $\gamma$, and enhance the infiltration of CD4+ and CD 8+ T cells in tumor microenvironment. Data are expressed as mean \pm SD. Each experimental group was compared to the control ( ${ }^{*} \mathrm{p} \leq 0.05 ;{ }^{* *} \mathrm{p} \leq 0.01 ;{ }^{* * *} \mathrm{p} \leq 0.001 ; \mathrm{ns}=$ not significant).
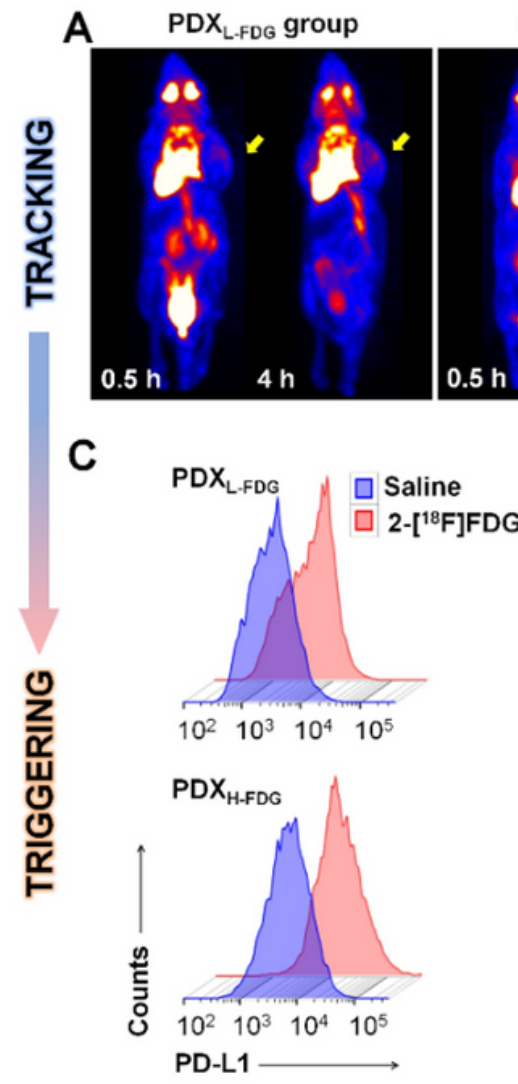

B

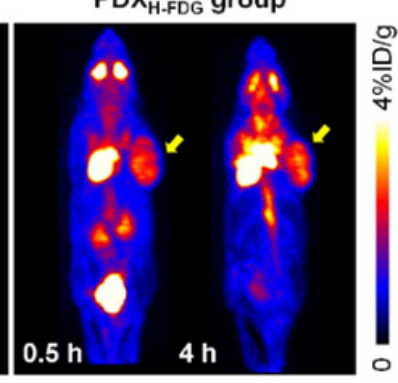

D

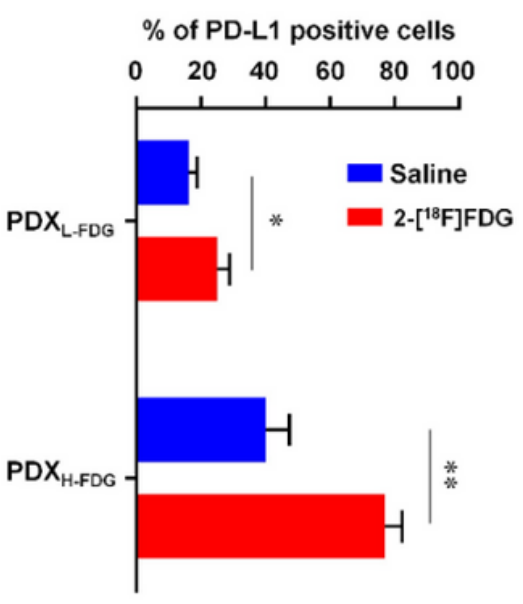

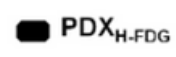

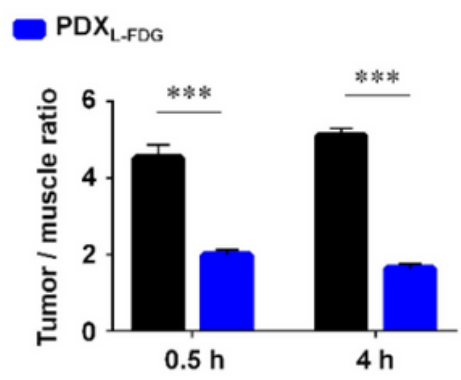

E

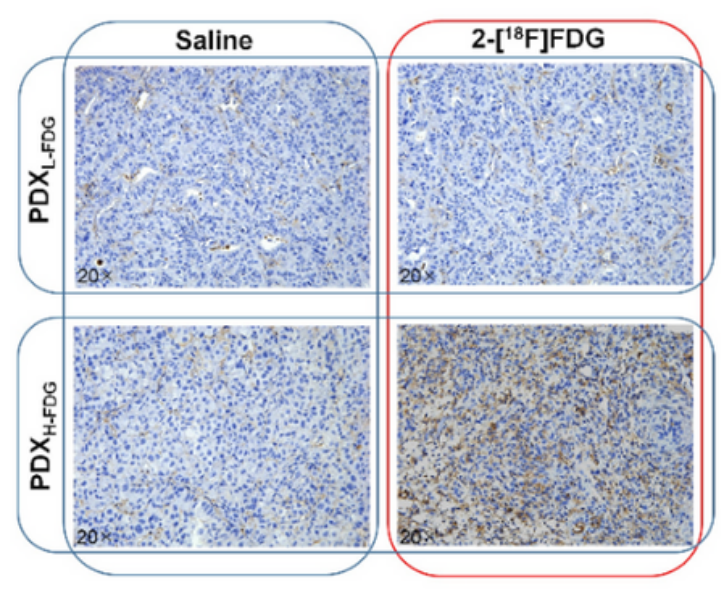

Figure 3

PD-L1 expression of PDX tumor was significantly increased after intravenous injection of 2-[18F]FDG. (A) Representative PET images of 2-[18F]FDG in PDX tumor models evaluated at $0.5 \mathrm{~h}$ and $4 \mathrm{~h}$ p.i. Tumor were indicated by yellow arrows. (B) Tumor uptakes (expressed in percentage injected dose per gram, $\% \mathrm{ID} / \mathrm{g}$ ) and T/M ratios derived from PET images by drawing regions of interest (ROIs). (C, D) Flow cytometry analysis of tumor PD-L1 expression at $4 \mathrm{~h}$ p.i. The tumor samples of PDX mice injected with saline were served as controls. There is an apparent difference in PD-L1 expression between 2-[18F]FDG and saline groups at $4 \mathrm{~h}$ p.i. Data are expressed as mean $\pm S D(n=3 /$ group $) .{ }^{*} p \leq 0.05 ;{ }^{* *} p \leq 0.01$; $* \star * p$ $\leq 0.001$. (E) IHC was performed to determine the PD-L1 expression on different tumor biopsies from PDXH-FDG and PDXL-FDG groups. Tumors were harvested at $4 \mathrm{~h}$ p.i. 

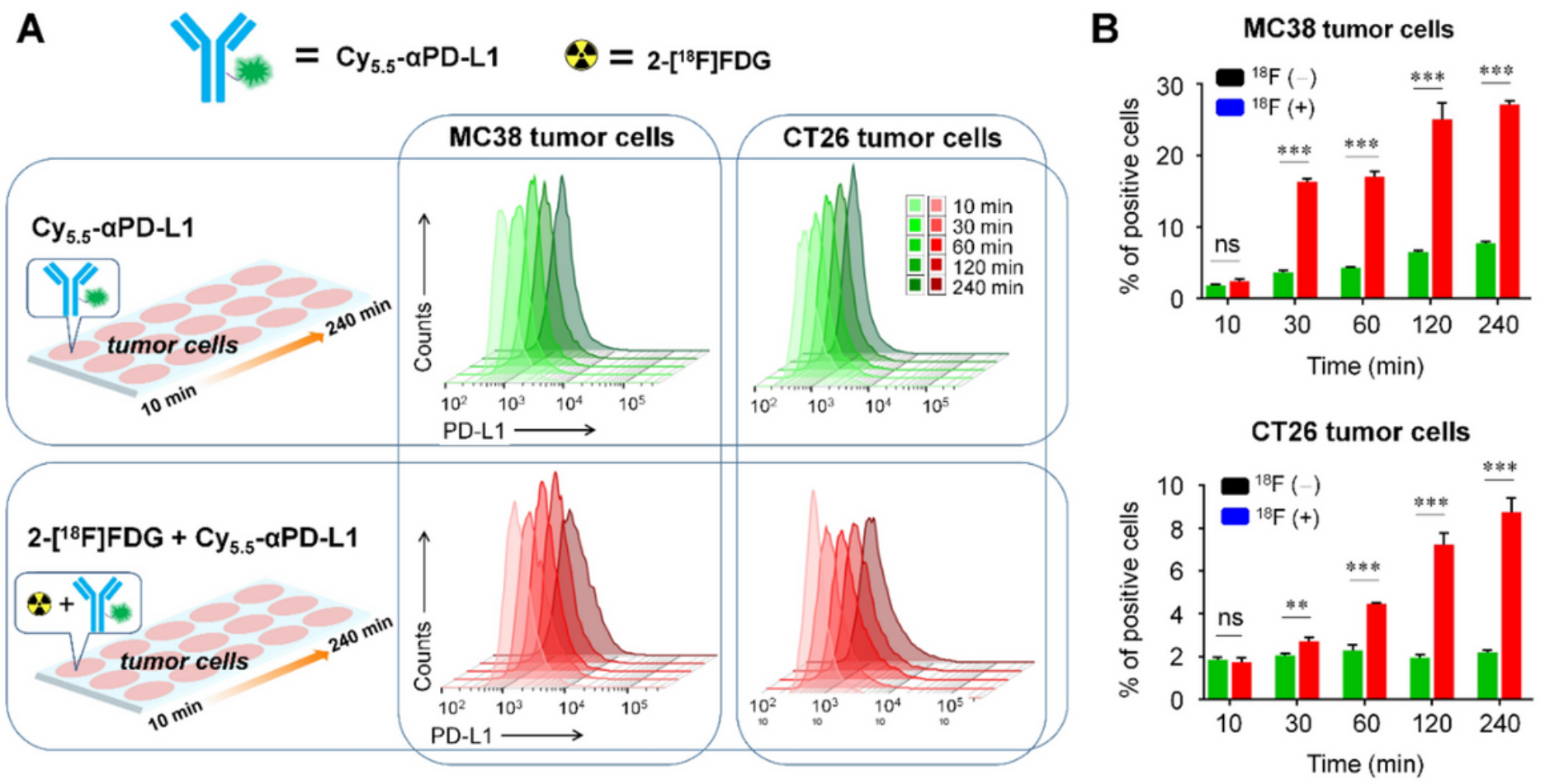

\section{Figure 4}

2-[18F]FDG causes increased APD-L1 mAb uptake by tumor cells. (A) The cell-binding properties of Cy5.5aPD-L1 on MC38 and CT26 cells were detected by flow cytometry. (B) The uptake of Cy5.5-PD-L1 was significantly increased after stimulation with 2-[18F]FDG in MC38 and CT26 cells. "(+)" in the figure legends refer to incubation with 2-[18F]FDG. ${ }^{*} \mathrm{p} \leq 0.05$; ${ }^{*} \mathrm{p} \leq 0.01$; ${ }^{\star \star *} \mathrm{p} \leq 0.001$; ns $=$ not significant. 

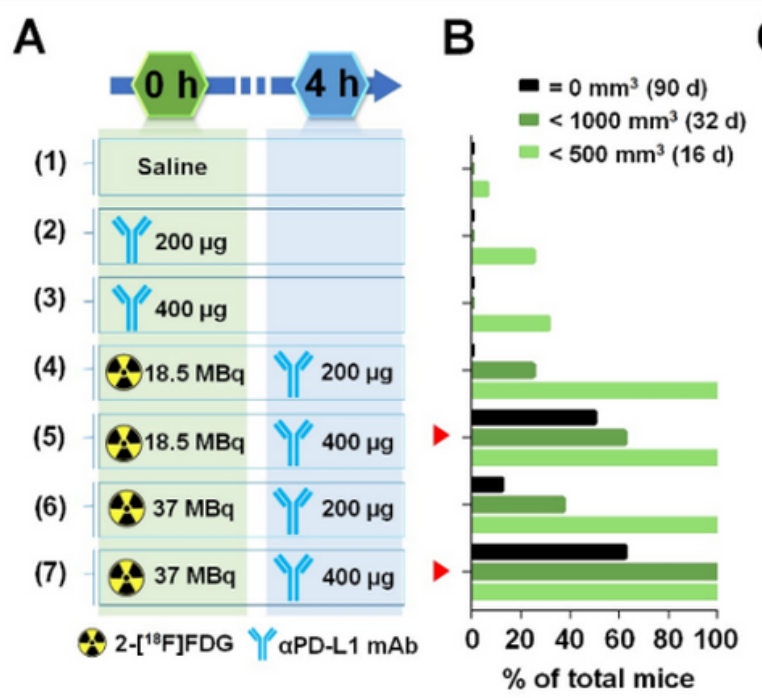

C
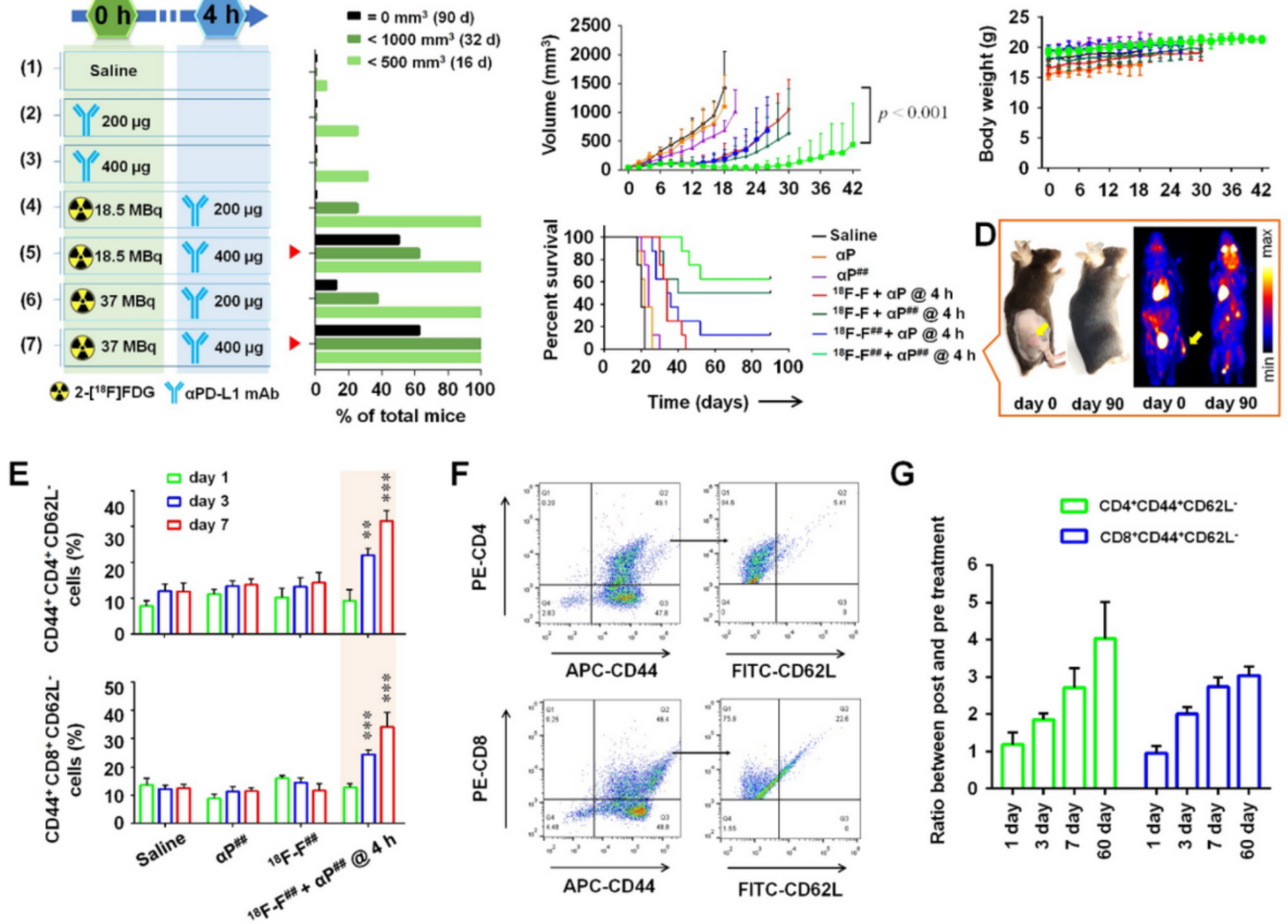

G
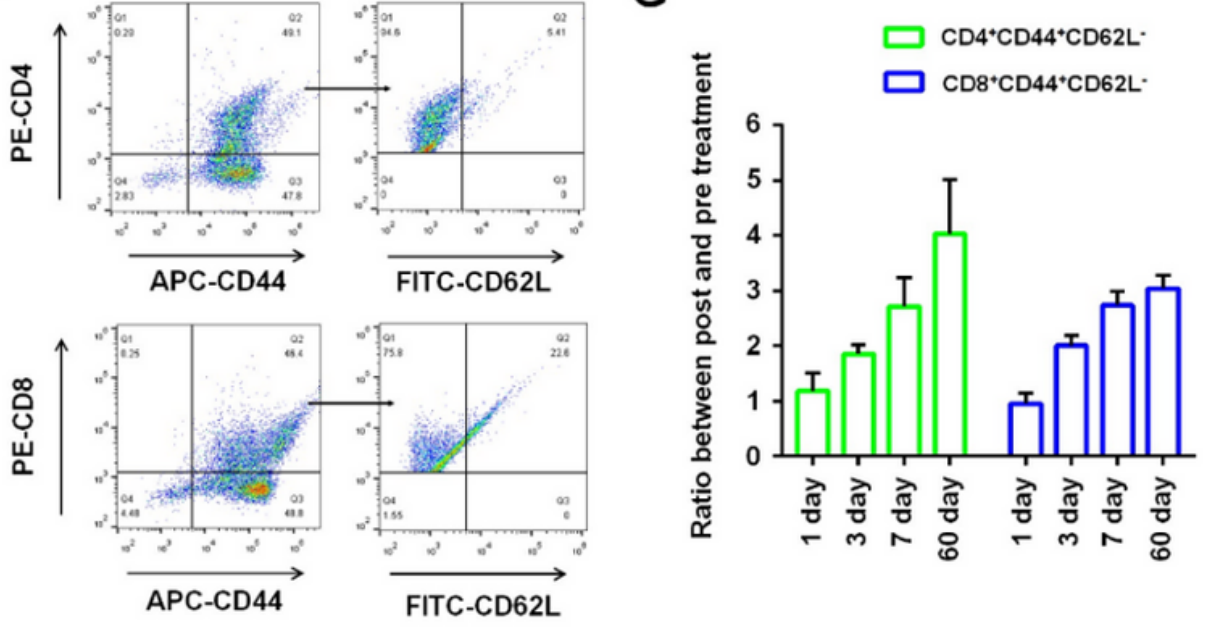

Figure 5

2-[18F]FDG-induced immunotherapy results in significant tumor growth delay and overall survival improvement. (A) Schematics of the procedures and timelines of treatment for MC38 tumor-bearing mice. (B) The percentage of tumor volumes under $500 \mathrm{~mm} 3$ on day $16,1000 \mathrm{~mm} 3$ on day 32 and $0 \mathrm{~mm} 3$ on day 90. (C) Time-dependent tumor growth curves, survival curves and weight changes of the MC38 tumor-bearing mice treated with 2-[18F]FDG induced immunotherapy. See Fig. S2A,B for more detailed information. Data are expressed as mean $\pm S D$ ( $n=8$ per group). Different doses of radiotracer and aPD$\mathrm{L} 1 \mathrm{mAb}$ were designated by the pound sign. Dose of aPD-L1 mAb: $10 \mathrm{mg} / \mathrm{kg} ; 20 \mathrm{mg} / \mathrm{kg}$ (\#\#). Dose of 2[18F]FDG: $462.5 \mathrm{MBq} / \mathrm{kg}$ (\#); $925 \mathrm{MBq} / \mathrm{kg} ; 1850 \mathrm{MBq} / \mathrm{kg}$ (\#\#). All radiotracers and aPD-L1 were administered by intravenous injection. (D) 2-[18F]FDG-PET imaging was performed on day 0 and day 90 to provide imaging reference for mice which were completely cured. Tumor sites were indicated by the yellow arrows. (E) Quantification of memory T cells (CD44+CD62L-) gating on CD8+ and CD4+ cells in the spleens of mice after different treatments on day 1, day 3 and day 7. See Fig. S4 for specific contents and quantitative indexes. Data are expressed as mean $\pm S D(n=3)$. Each experimental group was compared to the saline group ( $\left.{ }^{*} p \leq 0.05 ; * * p \leq 0.01 ; * \star * p \leq 0.001\right)$. (F) Representative flow cytometry plots of 
CD8+CD44+CD62L-and CD4+CD44+CD62L- memory T cells in the spleens of cured mice from 18F-F\#\#+ aP\#\# @ 4 h group on day 60. (G) The ratios of CD8+CD44+CD62L- and CD4+CD44+CD62L- memory T cells at different time points (day 1, 3, 7 and 60) after the implementation of 18F-F\#\#+ aP\#\# @ $4 \mathrm{~h}$ to those before treatment on day 0 .
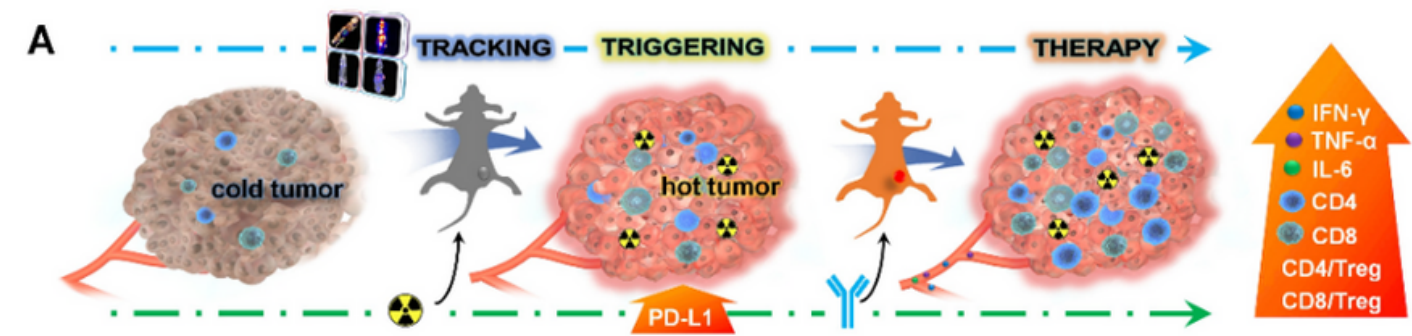

B
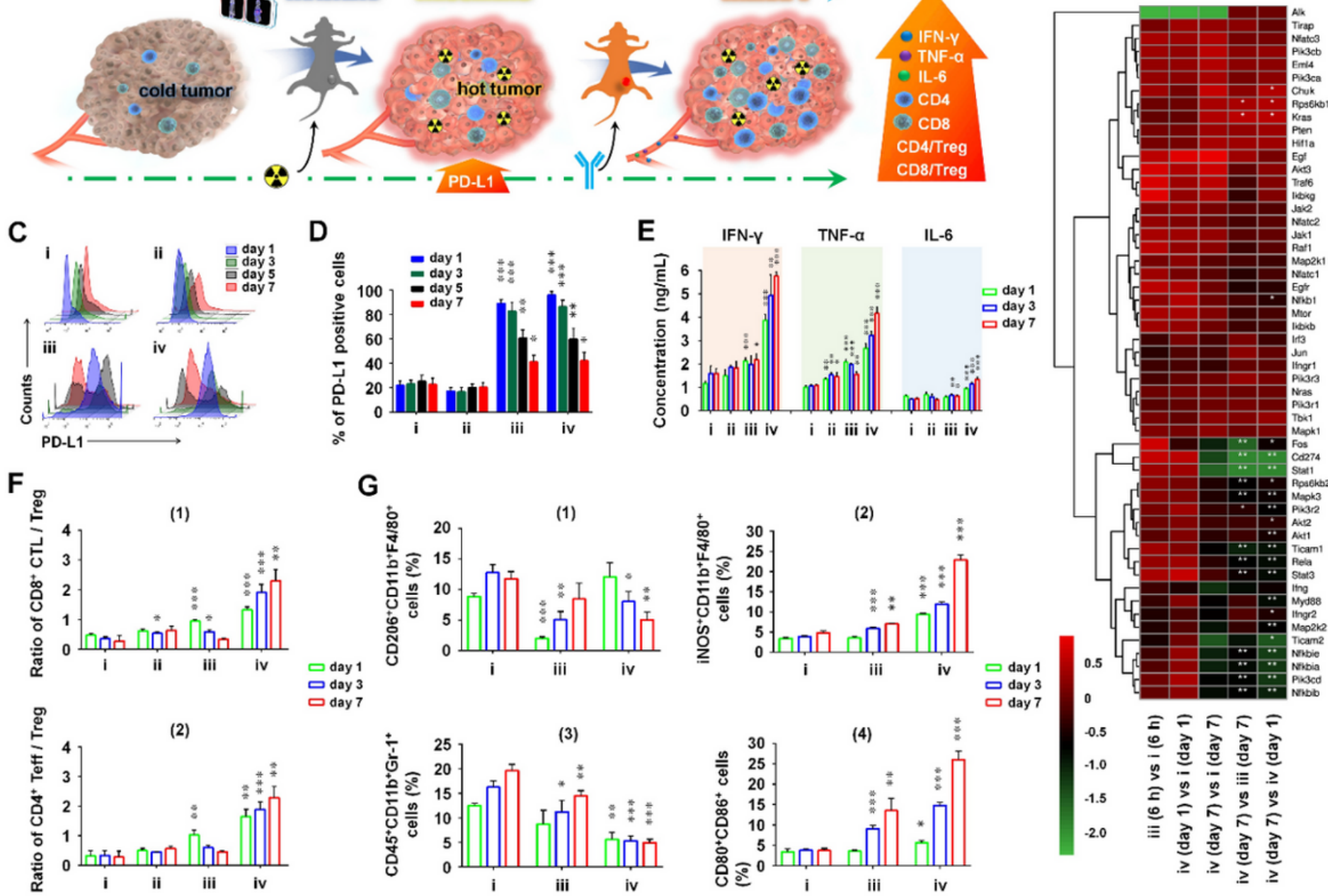

\section{Figure 6}

Response patterns of radiotracer-induced immunotherapy. (A) Schematics diagram of the possible mechanism for radiotracer-mediated antitumor immune response. Radiotracer in combination with aPDL1 mAb synergistically enhanced antitumor immunity by reprogramming TIME. (B) Heat map of DEGs associated with PD-L1 in MC38 tumor with different treatments and sampling time. The intensity of the color represents log2 fold-change. (C, D) The expression of PD-L1 in MC38 tumor treated with (i) saline, (ii) $400 \mu \mathrm{g}$ aPD-L1 mAb, (iii) $37 \mathrm{MBq}$ 2-[18F]FDG, and (iv) $37 \mathrm{MBq} 2$-[18F]FDG plus $400 \mu \mathrm{g}$ aPD-L1 mAb (4$\mathrm{h}$ interval). The tumors of different groups were collected on days 1, 3, 5 and 7 after the mice received different injections. (E) Dynamics of cytokine levels in the blood. The serum was separated and the concentrations of IFN- - , TNF- $a$ and IL- 6 were determined by ELISA. (F) Comparative analysis of CD8+ CTLs/Treg and CD4+ Th1/Treg in different groups. See Fig. S7A,B for specific contents and quantitative indexes. (G) The corresponding quantification of intratumoral total M2-like macrophages 
(CD206+CD11b+F4/80+), M1-like macrophages (iNOS+CD11b+F4/80+), MDSCs (CD45+CD11b+Gr-1+), and DCs (CD86+CD80+). See Fig. S8 for specific contents and quantitative indexes. Data are expressed as mean $\pm S D(n=3)$. Each experimental group was compared to the saline group $\left({ }^{\star} p \leq 0.05 ;{ }^{* \star} p \leq 0.01\right.$; $\star \star \star p \leq 0.001)$.

A

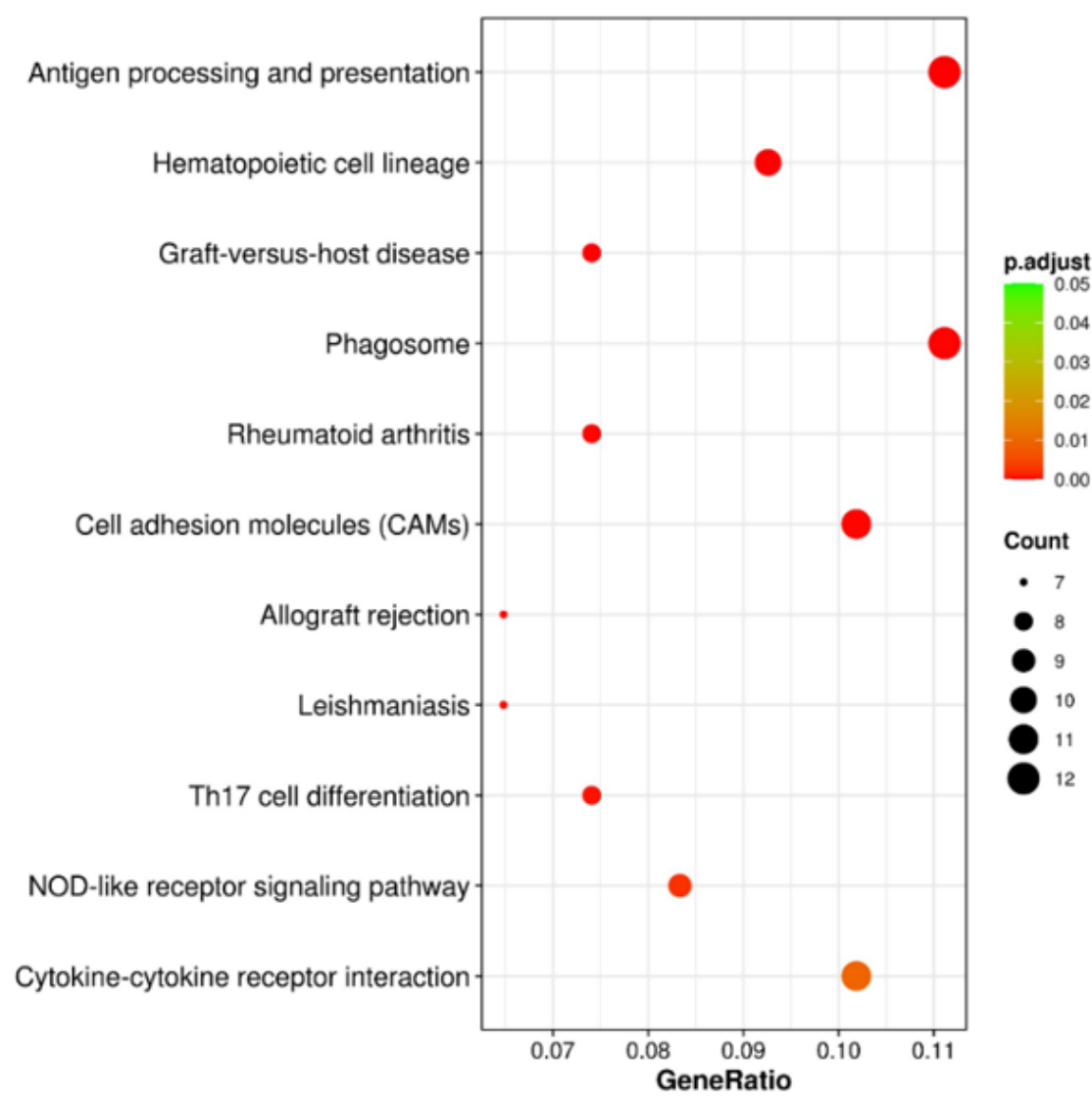

B

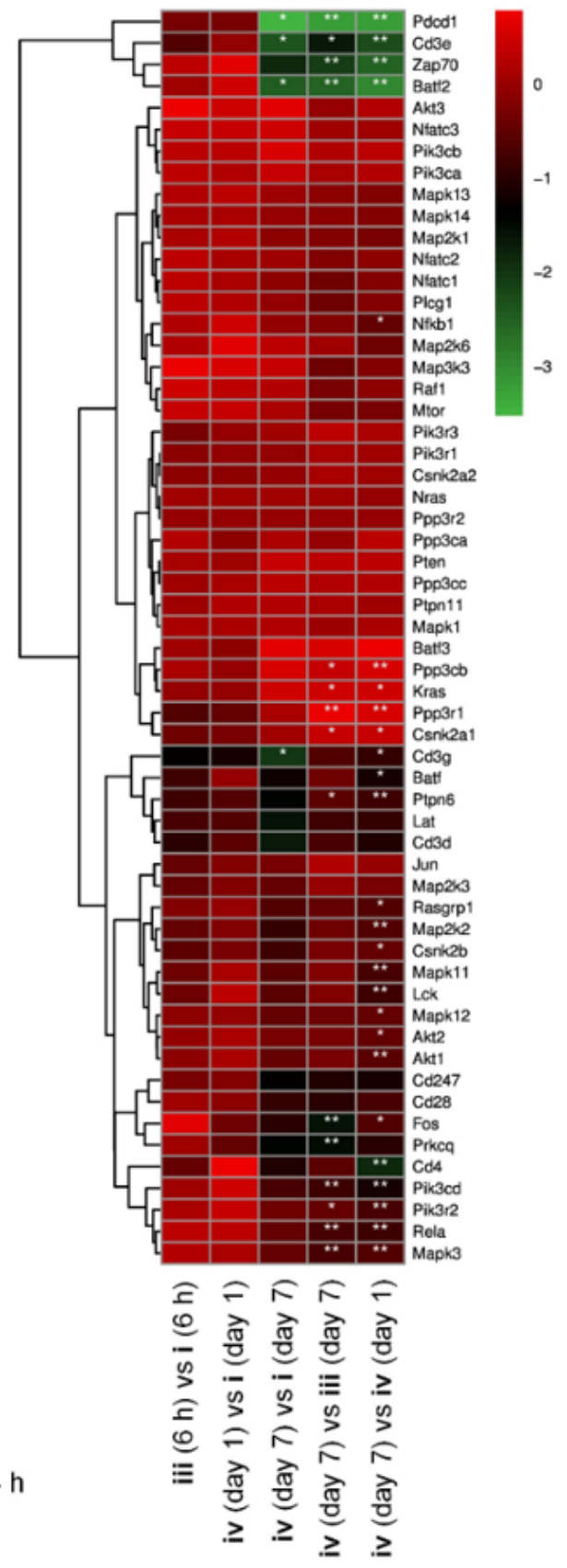

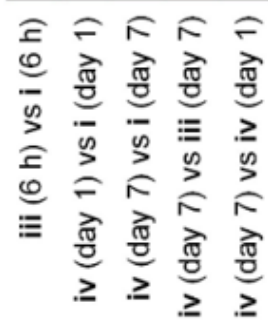

(i) saline

(iii) $37 \mathrm{MBq} 2-\left[{ }^{18} \mathrm{~F}\right] \mathrm{FDG}$

(iv) $37 \mathrm{MBq} 2-\left[{ }^{18} \mathrm{~F}\right] \mathrm{FDG}+400$ ug aPD-L1 @ $4 \mathrm{~h}$

\section{Figure 7}

Functional pathways and PD-1 changes during the 2-[18F]FDG-induced immunotherapy. (A) KEGG bubble map of the functional pathways involved in the biological effect of MC38 tumor induced by the therapeutic strategy of 37 MBq 2-[18F]FDG + 400 ug aPDL1 @ $4 \mathrm{~h}$. The sizes of the dots represent the counts of differential genes in the corresponding pathway. (B) Heat map of DEGs associated with PD-1 in 
MC38 tumor with different treatments and sampling time. The intensity of the color represents log2 foldchange.

\section{Supplementary Files}

This is a list of supplementary files associated with this preprint. Click to download.

- SupplementaryMaterials.docx

- zhideguoreportingsumm.pdf 